\title{
ER-Golgi and lysosomal pathways together drive fibrous tissue formation
}

\section{Adam Pickard}

University of Manchester

\section{Richa Garva}

University of Manchester https://orcid.org/0000-0001-7752-8936

\section{Antony Adamson}

University of Manchester https://orcid.org/0000-0002-5408-0013

\section{Ben Calverley}

University of Manchester

\section{Anna Hoyle}

University of Manchester https://orcid.org/0000-0002-6517-2546

\section{Christina Hayward}

University of Manchester https://orcid.org/0000-0003-3828-7126

\section{David Spiller}

University of Manchester

\section{Yinhui Lu}

Wellcome Trust Centre for Cell-Matrix Research

\section{Nigel Hodson}

University of Manchester

\section{Joe Swift}

Wellcome Centre for Cell-Matrix Research https://orcid.org/0000-0002-5039-9094

\section{Brian Bigger}

University of Manchester https://orcid.org/0000-0002-9708-1112

Karl Kadler ( $\nabla$ karl.kadler@manchester.ac.uk)

University of Manchester https://orcid.org/0000-0003-4977-4683

\section{Article}

Keywords: assembly, collagen, exocytic, extracellular matrix, fibroblasts, lysosomal storage, mucopolysaccharidosis, protein trafficking, secretion, SILAC, translation

Posted Date: March 3rd, 2022

DOI: https://doi.org/10.21203/rs.3.rs-1336021/v1 
License: (c) (i) This work is licensed under a Creative Commons Attribution 4.0 International License. Read Full License 
Adam Pickard ${ }^{1}$, Richa Garva ${ }^{1}$, Antony Adamson ${ }^{1}$, Ben C. Calverley ${ }^{1}$, Anna Hoyle ${ }^{1}$, Christina E. Hayward ${ }^{1}$, David Spiller ${ }^{1}$, Yinhui Lu ${ }^{1}$, Nigel Hodson ${ }^{2}$, Joe Swift ${ }^{1}$, Brian Bigger ${ }^{2}$ and Karl E. Kadler ${ }^{1 *}$ Wellcome Centre for Cell-Matrix Research ${ }^{1}$, Faculty of Biology, Medicine and Health ${ }^{1,2}$, University of Manchester, Manchester Academic Health Science Centre, Manchester, M13 9PT UK

* Corresponding author: karl.kadler@manchester.ac.uk

Keywords: Circadian rhythm; collagen; extracellular matrix; fibroblasts; homeostasis; protein trafficking; rhythmic; secretion; translation

9 Keywords: assembly, collagen; exocytic, extracellular matrix; fibroblasts; lysosomal storage, mucopolysaccharidosis, protein trafficking; secretion; SILAC, translation.

11

12

\section{SUMMARY}

Vast numbers of elongated collagen fibrils are assembled in the extracellular matrix during development then persist unchanged throughout life to maintain tissue shape. How cells distinguish between the synthesis of new fibrils and elongation of existing fibrils cannot be explained with our current understanding of protein secretion. Using CRISPR/Cas9 endogenous engineering of cells to express collagen-I containing photoswitchable and bioluminescent tags, we showed that fibroblasts secrete 100,000 collagen-I molecules/h via the conventional ER-Golgi pathway whereas fibril formation occurs at the plasma membrane at times longer than $24 \mathrm{~h}$ and requires functional lysosomes that store procollagen, without degradation, with a $t 1 / 2$ of $70 \mathrm{~h}$. We showed that cells from patients with lysosomal storage disorders exhibit impaired collagen fibril assembly. Thus, we have uncovered distinct transport routes for collagen fibril nucleation versus elongation. We suggest targeting lysosomal function could be a new approach for treating collagenopathies for which cures are unavailable.

\section{INTRODUCTION}

The ability of proteins to polymerize into elongated filaments, microfibrils and fibrils is essential for life. However, the mechanisms that determine how many assembly units (monomers, dimers or multimers depending on the protein) are used to initiate new polymers or grow pre-formed polymers are not fully understood. This is especially true for collagen fibrils, which are the most abundant protein polymer in vertebrates, accounting for $\sim 25 \%$ of total body protein mass (Smejkal and Fitzgerald, 2017). The fibrils are $D$-periodic (where $D \sim 67 \mathrm{~nm}$ ), can be centimeters in length (Craig et al., 1989), and range in diameter from $\sim 12 \mathrm{~nm}$ to $\sim 300 \mathrm{~nm}$ (Parry et al., 1978) depending on tissue and stage of development, and provide sites of attachment for a wide range of macromolecules including signaling complexes and cell receptors (Wickstrom and Fassler, 2011). In most tissues including skin, cornea, blood and lymphatic vessels, and musculoskeletal tissues, the fibrils occupy the vast majority of the tissue volume with large numbers of fibrils arranged in elaborate three-dimensional scaffolds that maintain tissue shape and mechanical integrity, even when the cells have been removed (e.g., see (Hodgson et al., 2018; Song et al., 2013)). The interdependence of the physiological requirements of the tissue and the number, size and arrangement of collagen fibrils means that a robust cellular mechanism must in place to regulate the nucleation of new fibrils or to grow existing fibrils. However, if and how cells distinguish between fibril nucleation and elongation cannot be explained by our current understanding of collagen synthesis. This decision is critical to tissue health because once 
42 formed, the fibrils are permanent and persist throughout life without being replaced (Heinemeier et 43 al., 2013; Heinemeier et al., 2016; Vaben et al., 2020).

44 Progress in understanding how cells regulate collagen fibril assembly has been hampered because 45 individual collagen fibrils are too narrow to be visualized by light microscopy, tagging strategies have not been developed to visualize fibril assembly in real time, and electron microscopy provides information only on pre-formed fibrils. Consequently, much of what we understand about collagen fibril assembly has come from in vitro studies using purified collagen. Collagen is readily solubilized from animal tissues into dilute acidic solution in which it remains stable for extended periods of time without unfolding or denaturation of the triple helix. Neutralization of these acidic solutions produces opaque gels containing $D$-periodic fibrils. This method of generating collagen fibrils was established in the 1950s (e.g. (Jackson and Fessler, 1955)) and continues to be widely used today to generate collagen fibrils in vitro. Fibrils can also be formed in vitro at neutral $\mathrm{pH}$ by cleaving the $\mathrm{N}$ - and $\mathrm{C}$ terminal propeptides of procollagen with ADAMTS2 and BMP-1, respectively (Kadler et al., 1990). Thus, there is compelling evidence that protein self-assembly is a major aspect of collagen fibril assembly. However, fibrils formed in vitro have no defined alignment, diameter, or length; thus, a motivation for our study was to understand how cells exert control over the self-assembly process in vivo.

Extensive cell biology and biochemistry studies have provided important insights into collagen synthesis. In brief, synthesis occurs by: (1) the folding of three polypeptide chains of procollagen (the soluble precursor) into a triple helix (Lamande and Bateman, 1999) in the endoplasmic reticulum (ER), followed by (2) transport of procollagen to the Golgi apparatus (Bonfanti et al., 1998; McCaughey et al., 2019) where cleavage of $\mathrm{N}$ - and C-propeptides can occur to generate mature collagen (Canty et al., 2004; Humphries et al., 2008; Stevenson et al., 2021), and (3) transport of procollagen and collagen in an undefined post-Golgi carrier to the plasma membrane (Polishchuk et al., 2000) for release to the extracellular matrix (ECM) where fibrils occur. Much is known about the accessory proteins and chaperones involved in procollagen folding (reviewed by (Ricard-Blum, 2011)) and transport of procollagen from ER to the Golgi (involving, for example, TANGO1 (e.g. see (McCaughey et al., 2021; Raote and Malhotra, 2021)) has been extensively studied but the molecular events leading up to fibril formation as procollagen progresses through the secretory pathway to the plasma membrane are less clear.

Glimpses into how cells assemble fibrils have come from electron microscopy studies of embryonic tendon and cornea, in which fibrils are seen to be attached via their pointed tips to the plasma membranes of cells (Birk and Trelstad, 1984; 1986; Canty et al., 2004; Kalson et al., 2013; Starborg et al., 2013; Trelstad and Hayashi, 1979; Young et al., 2014). Mathematical modelling based on electron microscopy images suggests that the fibrils grow by nucleation and propagation (Trotter et al., 2000) (analogous to the formation of crystals (Kadler et al., 1987)) in which the earliest-formed structures are $\sim 1 \mu \mathrm{m}$ in length (Graham et al., 2000) and are the precursors to the centimeter-long fibrils in adult tissues (Parry and Craig, 1977). Electron microscopy provides only a snapshot of the process and direct evidence for a cellular mechanism was lacking. A conundrum facing the cell-regulated model of fibril formation is that type I procollagen ( $\mathrm{PCl})$ is synthesized, partially or wholly converted to collagen within the cell, and then secreted to the extracellular space within $2 \mathrm{~h}$ (Canty et al., 2004; Mirigian et al., 2014). However, anecdotal evidence suggests that fibrils form at a much slower rate, and perhaps over the course of days. 
In this study, we used CRISPR/Cas9 gene editing of COL1A2 to endogenously tag the N-terminus of PCI with photoswitchable and quantifiable bioluminescent protein tags. This enabled us to image, at near super-resolution, collagen fibrils assembling at the plasma membrane. Correlative atomic force microscopy (AFM) confirmed that the fibrils had the characteristic $67 \mathrm{~nm} \mathrm{D-periodicity.} \mathrm{Combined}$ approaches involving photoswitching, immunofluorescence labeling, proteomics, and SILAC revealed newly-synthesized $\mathrm{PCl}$ transiting through a pool of pre-existing $\mathrm{PCl}$. This pool of stored $\mathrm{PCl}$ co-localized with procollagen chaperones (e.g. Hp47), fibril formation enhancers (e.g. procollagen C-proteinase enhancer (PCOLCE), lysyl hydroxylases (PLODs)), and, surprisingly, lysosomal markers (e.g. LAMP1, LAMP2, M6PR). No evidence of procollagen degradation was found. Furthermore, we showed that fibroblasts from patients with mucopolysaccharidosis lysosomal storage disorders exhibited an impaired ability to form fibrils but secretion of $\mathrm{PCl}$ was normal. These results showed that functional lysosomes are required for fibril formation but not secretion of $\mathrm{PCl}$, thereby showing separate intracellular pathways for fibril nucleation and fibril elongation. Our observations have important implications for tissue morphogenesis and highlight potential for cell targeted therapies aimed at regulating fibril assembly in combating diseases in which collagen synthesis and fibrous tissue formation are dysregulated.

\section{RESULTS}

Cells exhibit precise control of collagen fibril assembly

Using methods developed previously to enable quantitation of $\mathrm{PCl}$ in cultured cells (Calverley et al., 2020), we used CRISPR/Cas9 to introduce a nanoluciferase encoding sequence (Nluc) downstream of the signal peptide sequence of proa2(I) (encoded by Col1a2 and denoted Nluc-PCI). By comparing the amount of Nluc activity secreted into the culture medium to the levels of Nluc within cells we showed that fibroblasts secrete the equivalent of their entire collagen store in approximately $2.5 \mathrm{~h}$ (Figure 1A). Nluc-PCl could be detected in the medium within 5 min of changing the culture medium (Extended data figure 1A), in agreement with previous measurements of collagen secretion rates (Canty et al., 2004; Mirigian et al., 2014). To visualize the dynamics of PCl secretion we used CRISPR/Cas9 to introduce photoswitchable Dendra2 into proo2(I) at the same site used to introduce Nluc (Figure 1B and Extended data figure 1B-E). Insertion of Dendra2 did not disrupt the pro-fibrotic responsiveness of the cells (Figure S1F). Photoswitching of Dendra2-PCI from green to red allowed the fate of existing Dendra2-PCI to be traced Figure 1C, D, Extended data figure 1G, Video 1 ). The results showed that the photoswitched Dendra2- $\mathrm{PCl}$ had a half-life of $\sim 24 \mathrm{~h}$ which some Dendra2-PCl remaining the cell for a longer period (Figure 1D, red line). This estimate was slower than our previous estimate observed with the Nluc tag (Calverley et al., 2020) and implied that not all PCl is secreted rapidly. Using confocal microscopy with Airyscan detection, Dendra2-positive fibrils were conspicuous by their absence in the first day of culture, despite active collagen molecule secretion (Figure 1E). However, at $36 \mathrm{~h}$ in culture several Dendra2-positive fibrils began to appear at the same time (Figure 1F, Video 2). The time lag in appearance of the fibrils after extended time in culture was in line with previous studies that had detected type I collagen fibrils by immunofluorescence (Chang et al., 2020; Kubow et al., 2015).

Collagen fibril formation requires collagen to be in a triple helical confirmation (Kadler et al., 1988). Furthermore, pNcollagen (i.e., collagen with retained N-propeptides) readily assembles into fibrils in vitro and in vivo (Holmes et al., 1993; Hulmes et al., 1989). Therefore, we wanted to know if the fibrils formed from Dendra2-pNcollagen-I exhibited the characteristic $67 \mathrm{~nm} D$-periodicity. Using correlative Airyscan microscopy and atomic force microscopy (AFM) we showed that the Dendra2-positive fibrils 
exhibited a $D$-period of $65 \pm 7.4 \mathrm{~nm}$ and was within the expected range for collagen fibrils (Ushiki, 2002) (Figure 1G). In further experiments using an anti-collagen-I antibody we showed that the fibrils assembled by NIH3T3 fibroblasts were Dendra2 positive and recognized by an anti-collagen-I antibody (Extended data figure 2A). We engineered MC3T3 osteoblasts to express Dendra2-PCl and showed that the fibrils generated by these cells were Dendra2 positive and exhibited the characteristic $D$ banding of collagen fibrils (Extended data figure 2B, C). Taken together, these results showed that the Dendra2 tag had not disrupted procollagen secretion, procollagen folding or fibril assembly.

We noted that Dendra2-positive collagen fibrils were associated with cells; no fibrils were observed in isolation of the cells (Figure 2A, Video 3). We have observed that cells continually secrete collagen into the medium during $48 \mathrm{~h}$ (see Figure 1D); however, when we compared rates of Dendra2 signal loss from cells during the onset of fibril formation (here termed nucleation) to the loss of the red Dendra2 signal it was noted that cellular levels of Dendra2 diminished approximately 5 times faster during fibril nucleation (Figure 2B-C, Figure S2E). These data also show that levels of Dendra2 remain relatively stable prior to the onset of fibril formation and recover rapidly during fibril growth (Figure 2B, Figure S2D). From these experiments we postulated that cells can store collagen to achieve the high concentration required for nucleation (Kadler et al., 1987), but can also feed fibril growth by continued synthesis with prolonged culture (Figure S2F-H). Using a blue fluorescent protein with a KDEL retention signal to mark the ER we confirmed that the Dendra2-positive fibrils were deposited on the basal surface of cells (Figure 2D, Video 4). These experiments also identified loops within the Dendra2-positive fibrils that were located close to the BFP signal (Video 5). Finally, we generated 3D tissue mimetics in which fibroblasts grown in a fibrin gel under tension replace the fibrin with parallel fibrils of collagen (Kapacee et al., 2008). As shown in Extended data figure 2F-H, CRISPR-Cas9 edited $\mathrm{NIH} 3 \mathrm{~T} 3$ cells with endogenous knock-in of Nluc-PCl or Dendra2-PCl were highly effective at generating a matrix with aligned collagen fibrils.

\section{The extracellular environment regulates growth in length of fibrils}

To gain further insights into how cells generate collagen fibrils we used a bioreactor to pass a constant flow $(0.05 \mathrm{~mL} /$ minute) of culture medium over the apical surfaces of the cells, thereby removing newly-secreted collagen (shown schematically in Figure 3A). Assessment of Nluc levels showed that the flow reduced the levels of collagen in the medium by $99.5 \%$ compared to cells grown under static conditions (Figure 3B, C). Removing this secreted collagen had two significant effects: (i) the intracellular (Figure 3D) and (ii) extracellular matrix (Figure 3E) levels of collagen were reduced. Parallel experiments performed with Dendra2::Col1a2 edited cells showed that the numbers of collagen fibrils generated by cells did not alter between constant flow or static conditions (Figure 3F); however, the length of these fibrils were significantly shorter under constant flow (Figure 3G, H). These findings suggests that fibril nucleation occurs at the plasma membrane and the length of the fibril is influenced by extracellular environmental conditions.

\section{Contribution of extracellular collagen to fibril formation}

To explore further the concept that secreted collagen contributes to fibril elongation, we crossed the Col1a1fl/fl mouse (Li et al., 2017; Yang et al., 2013) with the Col1a2-CreERT(2) mouse (Li et al., 2017; Yang et al., 2013) and isolated tendon fibroblasts. These primary fibroblasts were then incubated with tamoxifen to stop endogenous full-length collagen production (Garva et al., manuscript in preparation). We then incubated these primary fibroblasts with conditioned medium from Dendra2:Col1a2 edited NIH3T3 cells. Using confocal microscopy with Airyscan processing we observed Dendra2-positive fibrils (Figure 4A). This not only confirmed our findings that cells control 
the fibril assembly process but also suggested that cells are able to internalize Dendra2-collagen for this purpose. This observation was confirmed by incubating primary human fibroblasts with Cy3labeled rat tail type I collagen (Extended data figure 3A, B); the Cy3-collagen was generated as previously described (Doyle, 2018). Tendon fibroblasts were effective at internalizing the Cy3-collagen in a time dependent manner (Figure 4B) and was confirmed in different cell types (Figure S3A-C). The uptake of collagen into cells was significantly enhanced in cells maintained in culture for prolonged periods and this further correlated with increased incorporation of Cy3-collagen into fibrils (Figure 4C). Using conditioned medium from Nluc:Col1a2 cells the dynamics of collagen uptake were monitored. The results showed that cells actively internalize Nluc- $\mathrm{PCl}$ in a concentration-dependent manner but reached a ceiling beyond which no more collagen was taken up (Figure 4D, Extended data figure 3D). This differed to the uptake of recombinant Nluc (rNluc) where cellular levels correlated directly with the amount of rNluc added to cells (Extended data figure 3E). The uptake of Nluc-PCI could also be further enhanced by the addition of increasing amounts of unlabeled rat tail collagen-I (Figure 4E). This process was also concentration limited suggesting that there are cellular processes actively involved in taking up the collagen. Addition of the dynamin/endocytosis inhibitor Dyngo4a to fibroblasts prior to addition of Nluc- $\mathrm{PCl}$ resulted in a reduced rate of Nluc- $\mathrm{PCl}$ internalization contributes to collagen fibrillogenesis, and that this process is enhanced with prolonged culture, helping to explain the lag period for collagen fibril assembly exhibited by cells.

193 Our previous studies using Nluc-PCl and pulse chase approaches (e.g., (Calverley et al., 2020; Canty et al., 2004)) focused on the fate of newly-synthesized procollagen. In the current study we were able to use the photoswitchable properties of Dendra2 to detect, simultaneously, (1) newly synthesized 'green' Dendra2-PCl, (2) pre-existing 'red' Dendra2-PCl, and (3) mixing of the two pools when the color changed to yellow. By analyzing time-lapse movies (Figure 5A and Video 1), we visualized green Dendra2-PCl transiting through the Golgi apparatus (Figure 5B) and then entering a pool of red Dendra2- $\mathrm{PCl}$, which then changed to yellow (Figure 5B and Extended data figure 4). Examination of the red channel showed pre-existing collagen fibrils and extracellular non-fibrillar red Dendra2-PCI.

To examine the rate of $\mathrm{PCl}$ secretion from unedited and Dendra2:Col1a2 edited fibroblasts, we introduced the HiBit split-nanoluciferase peptide sequence into the telopeptide region of the proa2(I) chain using CRISPR-Cas9 knock-in in the Col1a2 gene (see schematic in Figure 5C). Sampling of conditioned medium after addition of fresh culture medium indicated that Dendra2:HiBit:Col1a2 cells secreted collagen-I at the same rate as HiBit-PCI (Figure 5D). These experiments showed that the presence of the tags did not affect $\mathrm{PCl}$ secretion.

To examine further if fibroblasts are able to store PCl we grew unedited NIH3T3 fibroblasts for $48 \mathrm{~h}$ with $\mathrm{C}^{13}$-lysine and subjected intracellular proteins to analysis by liquid chromatography coupled tandem mass spectrometry (LC-MS/MS). The analysis was able to detect 'heavy' and 'light' peptides for 1380 proteins. Both 'heavy' and 'light' Col1a1-encoding peptides were detected after $48 \mathrm{~h}$ labeling of which 39-43\% of the peptides were 'light' (Figure 5E). Based on the abundance of heavy and light peptides the half-lives of the detected proteins were determined (Figure 5F). The presence of both 'heavy' and 'light' versions of peptides derived from proa1(I) identifies a pool of stable PCI (giving rise to the 'light' peptides) and a pool of $\mathrm{PCl}$ (giving rise to the 'heavy peptide') synthesized during the 48 $\mathrm{h}$ labeling with $\mathrm{C}^{13}$-lysine. The ratio of the light/heavy peptides was used to calculate the $t 1 / 2$ for 
proteins detected. This analysis showed that the $t \frac{1}{2}$ of 'light' proal(l) was $\sim 70 \mathrm{~h}$. For comparison,

218 Hnrnpa1 (involved in pre-mRNA packaging) had a t $1 / 2$ of $\sim 123 \mathrm{~h}$ and Lig1 (a DNA ligase) had a $\mathrm{t} 1 / 2$ of $\sim 34$ 219 h.

\section{Procollagen-I is transported in carriers that range in size and distribution}

221 By sampling different areas of large numbers of Dendra2:Col1a2 cells by confocal microscopy with 222 Airyscan detection, we were able to image pre-fibril forming events. Several features were apparent; 223 firstly, Dendra2-PCl occurred in a constellation of punctate compartments that ranged in size. 224 Secondly, we repeatedly observed looped structures derived from larger intracellular carriers that were apparent only when imaging in multiple z-planes (Figure 6A). In these large carriers, the collagen appeared to be restricted to the periphery and were close to sites of fibril assembly. During embryonic tendon development the tips of collagen fibrils are observed within plasma membrane fibripositors (Canty et al., 2004). Using volume-EM imaging and 3D-reconstruction of mouse tail tendon, we found that fibripositors were often in contact with ribbons of ER and electron dense vesicles typical of lysosomes (Figure 6B, Extended data figure 5A, Video 6).

\section{Procollagen-I localizes to ER and lysosomal compartments at sites of fibril assembly}

The next series of experiments tested the hypothesis that both ER and lysosomal-like compartments are involved in fibril formation. In tendon fibroblasts cultured for $72 \mathrm{~h}$, intracellular $\mathrm{PCl}$ localizes largely to the ER, as indicated by co-localization with the ER resident enzyme, protein disulfide isomerase (PDI) (Figure 6C). There were also regions where the two proteins did not co-localize. To determine the identity of $\mathrm{PCl}$-containing compartments we performed cellular fractionation followed by western blotting and LC-MS/MS protein identification. As expected $\mathrm{PCl}$ was identified in fractions that contained ER-resident protein (e.g. calreticulin, CALR). However, $\mathrm{PCl}$ was found in fractions that did not contain CALR (see fractions 5-7 in Figure 6D). Some non-CALR fractions contained Hp47, which binds triple helical collagen (Ishikawa and Bachinger, 2013) and lysosome associated membrane protein (LAMP)-1 (Figure 6D). These PCl-positive/CALR-negative fractions were analyzed by LCMS/MS to identify resident proteins. Pathway analysis of the proteins identified 'endocytosis' and 'lysosome' as the enriched pathways (Figure 6E). Peptides originating from LAMP1 and LAMP2 as well as core components of the retromer complex (Vps35, Vps26, and Vps29) and the sorting nexins SNX1 and 6 were present in $\mathrm{PCl}$-containing fractions (Supplementary table 1). Retromer is responsible for the recycling of transmembrane proteins to the cell surface or the trans-Golgi network (TGN) and prevents their degradation by the lysosomal system (Bonifacino and Hurley, 2008). Within these fractions, peptides along the length of both proa1(I) and proa2(I) were identified. Furthermore, peptides from both the $\mathrm{N}$ - and C-propeptides of $\mathrm{PCl}$ were detected (Figure 6F). Further analysis of the proteins within these fractions identified 35 of the known collagen-interacting proteins (Doan et al., 2019). These included Hp47 and PPIB that catalyzes peptidyl proline isomerization, which is the ratelimited step in folding of the collagen triple helix. Similarly, Colgalt1, which transfers of beta-galactose to the hydroxylysine residues on collagen, was also present. The collagen crosslinking enzymes, PLOD1-3, were also readily detected (Supplementary table 1). Taken together, the presence of these proteins suggests that these lysosomal compartments contain triple helical $\mathrm{PCl}$ molecules in preparation for fibril formation. 
compartments (Figure 7), as was Hp47 (Figure 7B and Extended data figure 6B post photoswitch). Dual-immunofluorescent staining confirmed collagen was present in the lysosomes of both mouse and human fibroblasts (Extended data figure 5B, C) and at sites close to collagen fibrils (Figure 7A, C). In a separate series of experiments, immunogold labeling electron microscopy of collagen-I in mouse tail tendon readily identified collagen in large diameter compartments, which are clearly distinguishable from ER (Figure 7D). Taken together, the results indicate that $\mathrm{PCl}$ is trafficked through lysosome-like compartments in vivo.

Next, we examined $\mathrm{PCl}$ secretion and fibril deposition in the presence of inhibitors of the protein secretion pathway and lysosome function. A previous study showed that the secretory pathway inhibitors brefeldin A and monensin (which inhibit ER-Golgi transport (Colanzi et al., 2013) and transport to lysosomes (Stenseth and Thyberg, 1989), respectively) inhibited Nluc-PCl secretion (Calverley et al., 2020). We confirmed here that the secretion of Nluc-PCl into the culture medium was highly sensitive to brefeldin A (Figure 8A). However, deposition of Nluc-positive collagen fibrils into the matrix over 3 days was unaffected (Figure $\mathbf{8 B}, \mathrm{C}$ ). This finding was confirmed when cultures of Dendra2:Col1a2 cells were grown for 3 days in the presence of brefeldin $A$, then decellularized and the amount of Nluc in the residual matrix measured. On the other hand, monensin almost completely blocked fibril formation (Figure 8D-F).

278 In the next series of experiments, we examined the effects of bafilomycin A1 and chloroquine on collagen fibril formation. These compounds impair lysosome function (reviewed by (Fedele and Proud, 2020)). Bafilomycin and chloroquine significantly inhibited fibril formation (Figure 8F and Extended data figure 7). Bafilomycin in combination with monensin completely blocked fibril formation (Figure $8 \mathrm{~F})$.

Further demonstration of the redundancy of the canonical secretory pathway in collagen fibril formation was shown by knockdown of the Golgi localized SNARE protein syntaxin 5 (Stx5). Stx5 is essential for ER-Golgi traffic of multiple cargoes including collagens (Nogueira et al., 2014). Here, knockdown of Stx 5 was effective in disrupting collagen secretion but had no impact on incorporation of collagen into the matrix (Figure 8G-J, Extended data figure 8).

Disrupted lysosome function results in defective deposition of type I collagen to the matrix Because inhibition of lysosome function results in reduced deposition of collagen to the matrix, we monitored type I collagen deposition in skin fibroblasts derived from patients with lysosomal storage disorders. Fibroblasts from patients with mucopolysaccharidosis (MPS) type I and type Illa were allowed to assemble a matrix during 3 days, after which we detected collagen fibrils by immunestaining using an anti-collagen I antibody. Levels of intracellular collagen did not appear to be overtly altered in MPS patient fibroblasts however, there was reduced deposition of collagen fibrils in these cultures. Failed fibril nucleation events were observed in fibroblasts from patients with both MPS type I and type IIIA MSP (red boxes), again implicating the lysosome as an essential compartment in the formation collagen fibrils (Figure 9A, B). CRISPR-Cas9 editing of lysosomal storage disorder patient fibroblasts with the split nanoluciferase tag allowed the secretion of endogenous $\mathrm{PCl}$ to be monitored. Secretion of HiBit-PCl molecules was unaffected despite defective collagen fibril formation. Previously, the ionophore ionomycin has been used in studies of lysosome exocytosis (Rodriguez et al., 1997). Here, we treated HiBit:Col1a2 cells with ionomycin and observed rapid release of HiBit-PCl from the fibroblasts (Figure 9C, D). 
305 In this report we show how lysosomes store collagen in preparation for collagen fibril assembly at the plasma membrane and that collagen secretion is not synonymous with collagen fibril formation. We show that the secretion of collagen monomers occurs via the conventional ER-Golgi secretory pathway whereas the assembly of collagen fibrils occurs via a lysosomal route. These findings help solve a longstanding puzzle of how cells traffic procollagen through the cell to either assemble a new fibril or to elongate one that has already been seeded. Understanding how cells assemble collagen fibrils (i.e., whether to nucleate new ones or grow existing ones) is important for developing strategies to control collagen homeostasis to combat a wide range of collagenopathies.

313 Our discovery that the processes of collagen secretion and collagen fibril assembly are independently 314 regulated stemmed from the observation that fibroblasts secrete 100,000 collagen molecules per $\mathrm{h}$ but fibril formation begins at least $24 \mathrm{~h}$ later. In the cell cultures used here, fibril assembly occurs on the underside of the cell where a microenvironment most probably exists to help concentrate extracellular collagen. This hypothesis is supported by our observation that once fibril assembly begins the process is completed swiftly. It is plausible that cells in vivo create a similar niche, perhaps between neighboring cells, to facilitate rapid propagation of fibril growth. The pathway of rapid collagen egress from the cell is clearly dependent upon traffic from ER to Golgi, as described elsewhere (Bonfanti et al., 1998). However, the involvement of compartments containing $\mathrm{PCl}$ and lysosomal proteins, such as LAMP1, to generate fibrils, was unexpected. Non-classical routes of secretion have been described for various cargoes, but these typically carry proteins without signal peptides (for review see (Kim et al., 2018)). Some Golgi-bypassing mechanisms have been described e.g., ER targeted cargoes such as alpha integrins, the thrombopoietin receptor, and mutant forms of CFTR (reviewed by (Kim et al., 2018)). The term 'secretory lysosome' has been used to describe dual function organelles that function both for degradation and storage of secretory proteins (reviewed by (Blott and Griffiths, 2002)). These secretory lysosomes are found in T cells, natural killer cells, mast cells and macrophages. Natural killer cells release toxic proteins by secretory lysosomes (reviewed by (van der Sluijs et al., 2013), and in cancer, secretory lysosomes can be a temporary store of immune checkpoint proteins (reviewed by (Wang et al., 2020)). Wounding of fibroblasts leads to the presentation of lysosomes to the cell periphery and is important to the repair of the cell membrane (Reddy et al., 2001). This presentation of lysosomes to the cell periphery is also observed during the differentiation of osteoblasts at times of enhanced collagen production (Beck et al., 2001; Nabavi et al., 2008). Thus, beyond the well documented role in protein degradation and recycling, lysosomal systems play a role in exocytosis for the purpose of cellular clearance and cell-cell communication (reviewed by (Buratta et al., 2020). In the context of collagen trafficking, the lysosome has typically been viewed as a degradative compartment via an autophagy pathway (Gorrell et al., 2021). We propose that fibroblasts use lysosomes to store and concentrate a pool of procollagen and collagen. Indeed, imaging approaches using Dendra2 showed the existence of the pool in the presence of active $\mathrm{PCl}$ synthesis and SILAC studies confirmed long-lived intracellular collagen with a $\mathrm{t} 1 / 2 \sim 70 \mathrm{~h}$. The lysosome is an ideal storage compartment for type I collagen due to its acidic $\mathrm{pH}$, which prevents spontaneous fibril formation in vitro (Harris and Reiber, 2007) at concentrations well above that needed for fibril nucleation (Kadler et al., 1990), and is a stable environment for the collagen triple helix which is highly resistant to proteolytic degradation, other than by neutral metalloproteinases. This storage function could represent a state of preparedness in the event of tissue damage or wounding. The rate of appearance of collagen in the lysosome compartment is slower than through 
the ER (Leblond, 1989) and others have demonstrated accumulation of collagen within the lysosome when applying high concentrations of inhibitors (Forrester et al., 2019). Recent studies following the trafficking of type I and II collagens in the presence of lysosomal inhibitors also suggest that collagen is trafficked to the lysosome (Forrester et al., 2019). Evidence from past studies suggest that not all collagen is rapidly secreted; labeling of newly synthesized collagen in rat fibroblasts appears in the lysosome at a slower rate than in the Golgi (Leblond, 1989). Together, these studies suggest that collagen may utilize this route during its exit from cells. In our present study the importance of the lysosome compartment in collagen fibril formation was demonstrated using lysosomal inhibitors and in fibroblasts derived from patients with lysosomal storage disorders, where lysosomal function is disrupted due to improper processing of glycoproteins. The defect in the ability to assemble an effective matrix may help to explain some of the skeletal and connective tissue malformations observed in these disorders. Importantly, these fibroblasts continue to secrete type-I collagen which again demonstrates the distinction between collagen secretion and fibril assembly.

Collagen concentration is critical to the dynamics of fibril formation in vitro, with a concentration of $0.4 \mu \mathrm{g} / \mathrm{mL}$ being needed to initiate fibril assembly (Kadler et al., 1987). We recently quantified the concentration of intracellular collagen (Calverley et al., 2020). In the main, intracellular collagen is below this nucleation limit but is exceeded in some intracellular vesicles. The high concentration of collagen within some vesicles implies that cells have the ability to store collagen. It is postulated here that acidification of these vesicles would be a simple method of preventing fibril formation within the cell. Our observations have important implications for the understanding of how fibrotic disease develop and highlight the potential for cell targeted therapies aimed at disrupting fibril assembly.

In conclusion, the use of CRISPR/Cas9 to tag collagen combined with absolute quantitation of collagen and super-resolution microscopy has allowed visualization of collagen fibril formation by living cells. The identification of separate transport routes for fibril nucleation and fibril propagation provides an explanation for how cells can regulate the number and growth of fibrils. The study also brings together extensive data on fibril formation in vitro and electron microscopy of fibrils in vivo into one unified model of cell-regulated nucleation-and-propagation to explain how cells build tissues from collagen fibrils. It is now tempting to suggest that diseases caused by aberrant matrix assembly and degradation could be treated by specific targeting of the two pathways.

\section{ACKNOWLEDGEMENTS}

378 KEK was supported by Wellcome (110126/Z/15/Z and 203128/Z/16/Z) and BBSRC (BB/T001984/1). JS

379 was funded by a Biotechnology and Biological Sciences Research Council (BBSRC) David Phillips 380 Fellowship (BB/L024551/1). BCC was supported by a Wellcome 4-year PhD studentship 381 (210062/Z/17/Z). The proteomics was performed at the Biological Mass Spectrometry Facility in the 382 Faculty of Biology, Medicine and Health (University of Manchester) with the assistance of Stacey 383 Warwood and Ronan O'Cualain, and electron microscopy was performed in the Electron Microscopy 384 Facility, Faculty of Biology, Medicine and Health (University of Manchester). We thank Kevin Kim 385 (University of Michigan, USA) and George Bou-Gharios (University of Liverpool) for providing mice. 386 The imaging of MPS fibroblasts was with the assistance of Joan Chang. B.B. was funded by the UK 387 MPS Society. The Bioimaging Facility microscopes used in this study were purchased with grants from 388 BBSRC, Wellcome, MRC and the University of Manchester Strategic Fund. 
390 KEK conceived the project. AA, AH, AP, BCC, CEH, DS, NH, RG, YL, performed experiments and collected 391 data. BCC performed the mathematical calculations. JS analyzed data. BB provided reagents and 392 obtained patient consent under REC 08H101063. All authors contributed to editing the manuscript 393 and support the conclusions. AP and KEK finalized the manuscript.

\section{DECLARATION OF INTERESTS}

395 The authors have no conflicting interests to declare.

396 ORCID IDs of authors

397 A.A.: http://orcid.org/0000-0002-5408-0013

398 A.H.: https://orcid.org/0000-0002-6517-2546

A.P.: http://orcid.org/0000-0001-9757-143X

400

B.B: https://orcid.org/0000-0002-9708-1112

401

B.C.C.: https://orcid.org/0000-0001-9403-0363

402

D.S.: https://orcid.org/0000-0003-2502-6787

403

C.E.H.: https://orcid.org/0000-0003-3828-7126

404

J.S.: https://orcid.org/0000-0002-5039-9094

405

K.E.K.: https://orcid.org/0000-0003-4977-4683

406

N.H.: https://orchid.org/0000-0002-7486-1976

407

R.G.: https://orcid.org/0000-0002-0588-7136

Y.L.: http://orcid.org/0000-0001-9034-0823

\section{FIGURE LEGENDS}

Figure 1: $\mathrm{PCl}$ secretion and collagen fibril assembly are separate processes.

412

A) Assessment of collagen secretion rate in CRISPR-edited Nluc::Col1a2 NIH3T3 cells. Nluc activity is readily detected in conditioned medium within minutes after medium change. Comparison to cellular Nluc::Col1a2 activity levels were used to assess the rate of type-I collagen secretion. $\mathrm{N}=4$, individual data points are shown.

B) CRISPR strategy to assess the lifetime of type I collagen intracellularly. Integration of the photoswitchable Dendra2 into the N-terminus of Col1a2, the short N-terminus of Col1a2 provides space within the N-pro-peptide for incorporation of Dendra2 into the type 1 collagen triple helix. Image modified from (Canty and Kadler, 2005).

C) Photoswitching of Dendra2::Col1a2 after 30 second exposure to $405 \mathrm{~nm}$ light.

D) Tracing of pre-existing Dendra2::Col1a2 (Red) pixel intensity demonstrating a slower rate of type-I collagen secretion, and a phased collagen production of new Dendra2::Col1a2, intensities are relative to the first frame after photoswitching (Red) or to the final frame (Green). The average pixel intensities of five cells are shown.

E) Dendra2::Col1a2 NIH3T3 cells grown on correlative grid coverslips imaged by widefield fluorescence microscopy, samples were then fixed to perform AFM of the same region. Assessment of the periodicity of the Dendra2 positive fibril demonstrated a D-Period of 65 $\mathrm{nm}$. 
F) Time-lapse Airyscan microscopy tracing Dendra2::Col1a2 NIH3T3 cells, $24 \mathrm{~h}$ after seeding no fibrillar structures were detected but with prolonged culture Dendra2 positive fibrillar structures were detected.

$\mathrm{G}$ ) Zoom of the cell imaged in $\mathrm{F}$ at the position of fibril formation. Scale bars represent $10 \mu \mathrm{m}$.

\section{Figure 2: Cells control collagen fibrillogenesis.}

A) Airyscan microscopy of Dendra2::Col1a2 NIH3T3 cells demonstrating that Dendra2 positive fibrils are formed within the boundary of the cell body. Images were captured after $48 \mathrm{~h}$ in culture. The maximum intensity projection of 51 different $Z$ positions are shown on the left, middle panel shows the localization of Dendra2::Col1a2 in a single Z plane within the cell body, right panel shows that Dendra2 fibrils are abundant on the cells basal surface, a single $Z$ plane is shown. Arrows indicate $Z$ position of each image. Video 2 shows all 51 positions.

B) Quantification of cellular Dendra2::Col1a2 signal intensity in the cell body prior to the onset of fibril formation, termed nucleation, cellular Dendra2::Col1a2 signals drop dramatically during nucleation but then later recover as fibrils continue to grow. Nucleation was set at the frame prior to the first detection of Dendra2 positive fibrils, this was designated as $T=0$, and the cellular Dendra2 signal was set as 1 , background pixel intensity was set as 0 . Data from five cells producing collagen fibrils are shown, data from individual cells are shown in Extended data figure 2. Average data points are shown, $n=5$ individual cells, error bars represent SD.

C) The loss of cellular Dendra2 signal observed in B demonstrates rapid release of cellular collagen at the onset of fibril formation, the rate of loss is compared to the rate of loss of photoswitched Dendra2 signal in the absence of fibril formation (Figure 1D). Average data points are shown, $\mathrm{n}=5$ individual cells, error bars represent SD.

D) Expression of BFP-KDEL in Dendra2::Col1a2 NIH3T3 cells demonstrates that fibrils are formed on the basal surface of cells. A maximum intensity projection of $42 \mathrm{z}$ positions is shown, left. A 3D reconstruction of these cells show collagen fibrils are distinct from the cell body (Video 3), however loops in the collagen fibrils were observed extending into the cell body, a 3D reconstruction is shown in Video 3. The middle panel shows that photoswitched Dendra2 positive fibrils are beneath the cell body, importantly at positions of where loops are engulfed by the cell the endoplasmic reticulum (BFP-KDEL) align with the fibrils.

Figure 3: Rapidly secreted collagen contributes to fibril growth but not fibril nucleation.

A) Schematic of CRISPR-Cas9 knock-in cells grown under constant flow.

B) To validate the flow system Nluc::Col1a2 NIH3T3 cells were grown under flow. Nluc activity was assessed in the medium within the Bioreactor housing cells seeded on glass coverslips. The activity was also measured in the medium within the waste outflow $(n=4$ independent experiments, error bars show SD).

C) Comparison of Nluc activity within the medium of Nluc::Col1a2 NIH3T3 cells grown under flow conditions or under static 'normal' culture conditions for $72 \mathrm{~h}$. Comparison of Nluc activity in the medium was compared to recombinant Nluc protein to estimate the concentration of collagen within the medium as described previously (Calverley et al., 2020).

D) After $72 \mathrm{~h}$ culture cells were removed from the bioreactor and subjected to assessment of cell viability and then coverslips were decellularized, measurements of Nluc activity in decellularized buffer were measured in triplicate $(n=4$ independent experiments, error bars show SD). 
E) After decellularization, Nluc activity associated with the deposited matrix by cell grown under flow measured relative to cells grown under static conditions. $\mathrm{N}=5$ independent experiments, error bars show SD.

F) The number of Dendra2 collagen fibrils per cell were scored, $n=3$ fields per view from 4 independent samples.

G) Dendra2::Col1a2 NIH3T3 cells were grown under flow or static conditions for $72 \mathrm{~h}$. Airyscan microscopy of live cells identified Dendra2 positive fibrils. Scale bar represents $20 \mu \mathrm{m}$ )

$\mathrm{H}$ ) The length of individual Dendra2 collagen fibrils were assessed using Image J image analysis software across all samples identified in G.

Figure 4: Endocytosed collagen contributes to fibril assembly.

Figure 5: Secretion and storage of $\mathrm{PCl}$.

A) Fibroblasts isolated from tamoxifen treated Col1a1 ${ }^{\mathrm{F} / F}$; Col1a2-ER ${ }^{\mathrm{t2}}$ mice (Li et al., 2017; Yang et al., 2013) were fed with conditioned medium from Dendra2::Col1a2 NIH3T3 for $72 \mathrm{~h}$, medium was replaced each day. Dendra2 positive fibrils were also detected associated with cells. Scale bar represents $10 \mu \mathrm{m}$.

B) $10 \mu \mathrm{g} / \mathrm{mL}$ Cy3 labeled rat tail collagen was added to cultures of human foreskin fibroblasts for 1 or $18 \mathrm{~h}$ demonstrating that fibroblasts were able to endocytose type-I collagen. Uptake was significantly enhanced in cells cultured for $72 \mathrm{~h}$ compared to those cultured for only $24 \mathrm{~h}$, see Extended data figure 3.

C) Human foreskin fibroblasts fed with $0.5 \mu \mathrm{g} / \mathrm{mL}$ Cy3 collagen for $18 \mathrm{~h}$ were effective at incorporating the labeled collagen into fibrillary structures whereas cells that were cultured for only $24 \mathrm{~h}$ were less effective in assembling fibrils.

D) Tendon fibroblasts were fed with conditioned medium from Nluc::Col1a2 NIH3T3 cells. The amount of Nluc activity taken up by tendon fibroblasts after $18 \mathrm{~h}$ was measured, demonstrating that cells were able to endocytose Nluc::Col1a2, however there was a limit to the amount of Nluc activity that can be endocytosed. $\mathrm{N}=3$ independent experiments, error bars show SD.

E) Tendon fibroblasts grown for $24 \mathrm{~h}$ were fed with Nluc::Col1a2 NIH3T3 conditioned medium along with increasing concentrations of rat tail collagen-I. After $18 \mathrm{~h}$ incubation, cellular Nluc activity was assessed. $\mathrm{N}=3$ independent experiments, error bars show SD.

\footnotetext{
A. Selected frames from Video 1 showing that photoswitched Dendra2::Col1a2 (Red) has a longer life span within cells than predicted from Nluc::Col1a2 edited cells. Photoswitched collagen was detected intracellularly after $30 \mathrm{~h}$ post-switch.

B. Selected frames from Extended data figure $\mathbf{4}$ showing transition of newly synthesized Dendra2::Col1a2 (displaying uniquely green fluorescence signal) through a Golgi-like structure. Concurrently pre-existing Dendra2::Col1a2 remained detectable beyond $9 \mathrm{~h}$ postphotoswitching.

C. CRISPR-Cas9 knock-in strategy to tag the amino telopeptide of proo2(I) with HiBit in unedited and Dendra2::Col1a2 cells within the region of Col1a2. HiBit is located C-terminal of the $\mathrm{N}$ proteinase cleavage site and $\mathrm{N}$-terminal of the start of the major triple helical domain. This site is encoded by sequences within exon 6 of the Col1a2 gene.

D. Introduction of the split nanoluciferase sequence encoding HiBit was confirmed by detection of HiBit in the medium of edited cells. The rate of secretion for HiBit::Col1a2 and
} 
Dendra2::Hibit::Col1a2 was measured as in Figure $1 \mathrm{~A}, \mathrm{n}=4$ replicate measurements, error bars shown SD.

E. To assess PCl lifetime in unedited NIH3T3 cells, cells were fed with $\mathrm{C}^{13}$ Lysine for $48 \mathrm{~h}$ before detachment, lysis and proteomic analysis. Peptide sequences shown are from the NC1 domain of proa1(I) mouse. Quantification of both heavy and light labeled Col1a1-derived peptides demonstrate that not all $\mathrm{PCl}$ is secreted within $48 \mathrm{~h}$. $\mathrm{N}=3$ independent experiments.

F. Global protein abundance in SILAC labeled NIH3T3 cells, the indicated protein half-lives were estimated by measuring the abundance of both heavy and light peptides for each protein as described in Methods.

Figure 6: $\mathrm{PCl}$ abundant at sites of fibril formation.

A. Dendra2::Col1a2 NIH3T3 imaged using Airyscan microscopy after $18 \mathrm{~h}$ after culture, 1000x magnification, and $2.5 \mathrm{x}$ digital zoom, a maximum intensity projection is shown. Vesicles containing Dendra2 signal are observed at sites of fibril assembly. Dendra2 signal was observed arranged at the periphery of vesicles, scale bar represents $1 \mu \mathrm{m}$.

\section{Figure 7: PCI localizes to LAMP1-positive compartments.}

B. Electron micrograph of embryonic mouse tail tendon cells containing fibripositors (red), individual frames taken from 3view reconstructions shown in Video 6, larger images are shown in Extended data figure 5. Fibripositors are regularly contacted by endoplasmic reticulum (blue) and electron dense vesicles (green). Video 7 show that the Golgi apparatus does not contact fibripositor.

C. Immunofluorescence imaging of type I collagen in mouse embryonic fibroblasts with the ER marker PDI. Scale bar represents $20 \mu \mathrm{m}$.

D. Fractionation of NIH3T3 using a sucrose density gradient, type I collagen is co-resident with the ER protein Calreticulin (Calr) but also with the lysosomal protein Lamp1 and the collagen chaperone Hp47.

E. Proteomic analysis of lysosomal fractions 6 and 7 identified significant enrichment of proteins identified by proteomic analysis of fractions 6 and 7 based on GO Cellular component terms.

F. Col1a1 (Red and Magenta) and Col1a2 (Blue and Green) derived procollagen peptides were present within both fractions 6 and 7 suggesting that newly synthesized collagen transitions these compartments.

A. Dendra2::Col1a2 NIH3T3 imaged using Airyscan microscopy $48 \mathrm{~h}$ after transfection with LAMP1-YFP. 1000x magnification, and 2.5x digital zoom, a maximum intensity projection of 31 images is shown. LAMP1-YFP vesicles containing photoswitched Dendra2 signals are observed. Three LAMP1-positive areas are enlarged. Using a single $Z$ plane the pixel intensity of Dendra2 (Red) and LAMP1-YFP was measured in FIJI. At sites of fibril assembly LAMP1-YFP was observed juxtaposed to red Dendra2 fibrils. Scale bar represents $20 \mu \mathrm{m}$.

B. Live cell super resolution microscopy of NIH3T3 stably transduced with Hp47-BFP-RDEL lentivirus and transfected with LAMP1-YFP. Scale bar represents $20 \mu \mathrm{m}$.

C. Immortalized tail tendon fibroblasts were grown in ascorbic acid containing medium for 3 days, cells were fixed and permeabilized and stained for collagen I, LAMP1 and counter stained with DAPI. A single optical section showing collagen I localization to LAMP1-positive lysosomes, a type I collagen fibril is indicated by the white arrow. Pixel intensity across the LAMP1-positive vesicle is shown. 
D. Immunogold electron microscopy labelling of collagen-1 of mouse tendon identifying extracellular collagen fibrils and intracellular collagen within hollow lumen vesicles, left, individual vesicles are enlarged. Scale bar represents $1 \mu \mathrm{m}$.

\section{Figure 8: Non-classical PCl secretion feeds fibril assembly.}

A. Schematic for monitoring Nluc activity secretion from Nluc::Col1a2 NIH3T3 cells. Comparison of Nluc secretion rates in control cultures and cells treated with $100 \mathrm{nM}$ brefeldin A identified robust reduction of Nluc activity secretion.

B. Schematic to quantify the deposition of Nluc::Col1a2 into the extracellular matrix.

C. Nluc::Col1a2 NIH3T3 cells were cultured with $100 \mathrm{nM}$ brefeldin A for $72 \mathrm{~h}$, Nluc activity levels in the conditioned medium, cellular fraction and matrix were assessed. $\mathrm{N}=3$ independent experiments, $\mathrm{N}=4$ technical repeats from a representative experiment are shown. Error bars represent standard deviation.

D. Representative Airyscan confocal microscopy images of Dendra2::Col1a2 NIH3T3 cells cultured for $72 \mathrm{~h}$ in the presence of either $100 \mathrm{nM}$ brefeldin $\mathrm{A}$, or $1 \mu \mathrm{M}$ monensin with or without addition of the lysosome proton pump inhibitor $1 \mathrm{nM}$ bafilomycin. Dendra2 signals are shown to highlight deposited fibrils. Maximum intensity projections of $5 \mathrm{z}$ planes are shown. Scale bar represents $50 \mu \mathrm{m}$. Boxes are enlarged in $\mathrm{E}$.

E. Enlargements of images within the regions highlighted in D.

F. Quantification of Dendra2 fibril numbers per cell, $n=5$ independent experiments for brefeldin and monensin treatment, with an additional $n=2$ independent experiments for treatments including bafilomycin treatment. A minimum of 200 cells per condition, per experiment, were score. Error bars represent SEM. ${ }^{* *}$ represents $p<0.01$, Student's T-test, unpaired.

G. Western blot of Syntaxin 5 (Stx5) knockdown with 100 pmol siRNA in NIH3T3 cells. $\mathrm{N}=3$ independent experiments.

H. Syntaxin 5 (Stx5) knockdown with 100 pmol siRNA in Dendra2::Col1a2 NIH3T3 cells were cultured for $72 \mathrm{~h}$ before live imaging of Dendra2 fibrils. . Maximum intensity projections of 5 $z$ planes are shown. Scale bar represents $50 \mu \mathrm{m}$.

I. Quantification of Dendra2 fibril numbers per cell in siRNA treated Dendra2::Col1a2 NIH3T3. $\mathrm{n}=2$ independent. Error bars represent SD.

J. Syntaxin 5 (Stx5) knockdown with 100 pmol siRNA in Nluc::Col1a2 NIH3T3 cells were cultured for $72 \mathrm{~h}$, Nluc activity levels in the conditioned medium, cellular fraction and matrix were assessed. $\mathrm{N}=3$ independent experiments, each with $\mathrm{N}=4$ technical repeats are shown for conditioned medium and cellular fractions, or $n=2$ technical repeats for matrix measurements. Error bars represent standard deviation.

Figure 9: Disruption of type-I collagen fibril assembly in fibroblasts from patients with lysosome storage disorders.

A) Immunofluorescence detection of type-I collagen in human skin fibroblasts taken from patients with lysosomal storage disorders, mucopolysaccharidosis type 1 and tpe 3a (MPSI, and MPSIIIa). Scale bars represent $20 \mu \mathrm{m}$.

B) Regions highlighted in A show failed collagen assembly sites in MPS patient derived fibroblasts.

C) CRISPR-Cas9 mediated knock-in of the split nanoluciferase tag, Hibit, into the Exon 1 of Col1a2 in control HFF and MPS patient derived fibroblasts. Secretion rates of Hibit tagged collagen 
were normalized to cellular Hibit levels and indicated that there was no difference in the ability to secrete type-I collagen.

D) lonomycin triggered release of Nluc::Col1a2 from NIH3T3 cells. $\mathrm{N}=9$ independent experiments, error bars represent SEM.

\section{EXTENDED DATA FIGURES}

Extended data figure 1: $\mathrm{PCl}$ secretion and collagen fibril assembly are separate processes.

A) Statistical analysis comparing nanoluciferase activity detected in fresh culture medium applied to Nluc::Col1a2 NIH3T3 cells. $n=4$ at each time point. Levels in the culture medium were compared to the levels of Nluc activity in cells. *** represents Paired Student's T-test, $\mathrm{p}<0.001$. Error bars represent SD.

B) CRISPR-Cas9 knock-in strategy to integrate Dendra2 encoding sequences into the Col1a2 gene. A guide RNA immediately downstream of the signal peptide of the proa2(I) chain, directing Cas9 to exon 1 of Col1a2. A repair template encoding the Dendra2 coding sequence flanked by 800 bp homology arms.

C) PCR validation of Dendra2 integration into the Col1a2 gene. DNA was extracted from a single cell clone identified as Dendra2 positive by FACS. Primers for PCR are as shown in B.

D) Western blot validation of Dendra2 knock-in in the same single cell clone compared with unedited NIH3T3.

E) Real-time PCR primers were designed to detect Dendra2 knock-in, PCR products we subjected to Sanger sequencing.

F) The edited Dendra2:Col1a2 locus retrained responsiveness to TGF- $\beta$ I treatment and was induced transcriptionally following treatment with $2.5 \mathrm{ng} / \mathrm{mL}$ TGF- $\beta$ I for $48 \mathrm{~h}$, additional TGF$\beta$ I responsive transcripts were also measured. $\mathrm{N}=3$ independent repeats. Error bars represent the SEM.

G) Airyscan microscopy of Dendra2:Col1a2 NIH3T3 cells demonstrating that Dendra2 positive fibrils are formed within the boundary of the cell body. Images were captured after $43 \mathrm{~h}$ in culture. The maximum intensity projection of five images taken a different $Z$ positions are shown.

Extended data figure 2: Rapid release of intracellular collagen at the time of fibril formation.

A) Immunofluorescent detection of Dendra2 and collagen in Dendra2::Col1a2 NIH3T3 cells grown for 3 days after passage. Dendra2 positive fibrils are also positive for type-I collagen. Image captured at 1000x magnification. Scale bar represents $20 \mu \mathrm{m}$.

B) Airyscan microscopy of Dendra2:Col1a2 edited MC3T3 cells captured at 40x magnification, 41 $\mathrm{h}$ after seeding. Arrows indicate Dendra2 positive fibrils deposited by cells. Scale bar represents $20 \mu \mathrm{m}$.

C) Electron micrograph of MC3T3 cells and Dendra2:Col1a2 edited MC3T3 cells focusing on the collagen fibrils formed beneath the cells.

D) Quantification of cellular Dendra2:Col1a2 signal intensity in the cell body prior to the onset of fibril formation, termed nucleation, cellular Dendra2::Col1a2 signals drop dramatically during nucleation but then later recover as fibrils continue to grow. Nucleation was set at the frame prior to the first detection of Dendra2 positive fibrils, this was designated as $T=0$, and the 
cellular Dendra2 signal was set as 1 , background pixel intensity was set as 0 . Data from individual cells are shown, the average data is shown in Figure 2B.

E) The loss of cellular Dendra2 signal observed in Figure2C demonstrates rapid release of cellular collagen at the onset of fibril formation. The rate of loss is compared to the rate of loss of photoswitched Dendra2 signal in the absence of fibril formation (Figure 1D). $n=5$ individual cells, error bars represent SD.

F) 200,000 Nluc:Col1a2 NIH3T3 or Dendra2::Col1a2 NIH3T3 cells were grown as uniaxial 3D constructs.

G) Deposition of Dendra2::Col1a2 collagen fibrils orientated in the direction of mechanical tension imaged after 7 days in culture $(n=4)$.

H) Nluc:Col1a2 NIH3T3 constructs grown until Day 5 when medium was changed to monitor the secretion of Nluc activity into the medium over $48 \mathrm{~h}$. A constant rate of Nluc secretion was observed ( $n=3$ independent experiments, each conducted with $n=4$ technical replicates for a representative experiment is shown, error bars represent SD).

\section{Extended data figure 3: Endocytosed collagen contributes to fibril assembly.}

A) $10 \mu \mathrm{g} / \mathrm{mL}$ Cy3 labeled rat tail collagen was added to cultures of human lung fibroblasts for 1 or $18 \mathrm{~h}$ demonstrating that fibroblasts were able to endocytose type-I collagen. Uptake was significantly enhanced in cells cultured for $72 \mathrm{~h}$ compared to those cultured for only $24 \mathrm{~h}$.

B) Quantification of Cy3 positive cells after exposure to $10 \mu \mathrm{g} / \mathrm{mL}$ Cy3 labeled rat tail collagen added to human foreskin fibroblasts $n=3$ independent experiments, error bars represent SEM.

C) Assessment of Cy3 labeled rat tail collagen uptake into SAOS2 cells for $1 \mathrm{~h}$, demonstrating that these cells are able to endocytose type-I collagen with increased uptake with prolonged culture.

D) NIH3T3 fibroblasts were fed with conditioned medium from Nluc:Col1a2 NIH3T3 cells. The amount of Nluc activity taken up by tendon fibroblasts after $18 \mathrm{~h}$ was measured, demonstrating that cells were able to endocytose Nluc:Col1a2, however there was a limit to the amount of Nluc activity taken that can be endocytosed. $\mathrm{N}=3$ independent experiments, error bars show SD.

E) Tendon fibroblasts were fed with conditioned medium from NIH3T3 cells supplemented with varying concentrations of recombinant Nluc (rNLuc). The amount of Nluc activity taken up by tendon fibroblasts after $18 \mathrm{~h}$ was measured, the amount of Nluc activity taken up was directly related to the amount of rNLuc added to the medium. $\mathrm{N}=3$ independent experiments, error bars show SD.

F) NIH3T3 cells were seeded in $35 \mathrm{~mm}$ dishes and loaded with the live cell nanoluciferase substrate, endurazine for $2 \mathrm{~h}$, cells were then treated with DMSO or $10 \mu \mathrm{M}$ Dyngo4a. Luminescence was then measured every 10 minutes after addition of $1 \mathrm{~mL}$ of NLuc::Col1a2 conditioned medium cell metabolized substrate. Representative traces are shown. $\mathrm{N}=3$ independent experiments.

Extended data figure 4: Secretion of newly synthesized PCI with concomitant storage of PCI.

Dendra2::Col1a2 NIH3T3 cells were photoswitched at $\mathrm{T}=0$. Merge of both green and red fluorescence from Dendra2. Green Dendra2::Col1a2 is first observed at $2.5 \mathrm{~h}$ post-photoswitch. After $3 \mathrm{~h}$ uniquely green fluorescent protein (newly synthesized Dendra2:Col1a2) is detected in a Golgi-like organelle. Newly synthesized Dendra2:Col1a2 continues to be detected for a further $2 \mathrm{~h}$ but then is distinctly 
absent in subsequent frames, implying secretion. Meanwhile, photoswitched Dendra2:Col1a2 remains present within the cell and is suggestive of a retained cellular pool of type-I collagen.

Extended data figure 5: Fibripositors are regularly contacted by endoplasmic reticulum and electron dense vesicles.

A. Individual frames taken from Video 6 demonstrating that a single fibripositor is contacted regularly by both endoplasmic reticulum and electron dense vesicles. Areas highlighted by red boxes are used in Figure 6B.

B. Confocal images of type I collagen and LAMP1 indirect immunofluorescence staining in mouse tendon fibroblasts. Scale bar represents $20 \mu \mathrm{m}$.

C. Confocal images of type I collagen and LAMP1 indirect immunofluorescence staining in human foreskin fibroblasts. Scale bar represents $20 \mu \mathrm{m}$.

\section{Extended data figure 6: $\mathrm{PCl}$ localized in lysosomes.}

A. On gridded $35 \mathrm{~mm}$ dishes Dendra2::Col1a2 $3 T 3$ cells were transfected with LAMP1-YFP overnight with Fugene 6, cells were then grown in full medium with ascorbic acid for $48 \mathrm{~h}$. LAMP1-YFP positive cells were then photoswitched for $30 \mathrm{sec}$ on a Nikon Eclipse microscope.

B. LAMP1-negative cells (Region 1) and LAMP1-positive cells (Region 2) were imaged with Zeiss Airyscan 880 in super-resolution mode. Photoswitched Dendra2-Col1a2 fibrils can be observed in Region 1 demonstrated by the detection of a red fluorescence signal. In a neighboring LAMP1-YFP positive cell, Region 2 the photoswitched Dendra2-PCl signal is located within the lumen of the lysosome. The highlighted region 2 shows the area magnified in Figure 7A. Scale bar represents $10 \mu \mathrm{m}$.

\section{Extended data figure 7: Non-classical PCl secretion feeds fibril assembly.}

A) Cell viability as assessed by Prestoblue metabolism after $72 \mathrm{~h}$ treatment of tendon fibroblasts with increasing doses of chloroquine (Blue) and bafilomycin (purple), viability to compared to equivalent doses of DMSO (black).

B) Confocal microscopy of immunofluorescent detection of type-I collagen in DMSO, chloroquine and bafilomycin treated tendon fibroblasts, upper. Enlarged regions showing type-I collagen fibrils, lower.

C) Quantification of type-I collagen fibrils per cell, $n=4$ independent experiments, at least 2000 cells were scored per treatment. Error bars represent SEM.

D) Measurement of type-I collagen fibril length. ${ }^{* * *}$ represents $p<0.001$, Students T-test, unpaired. Error bars represent SD.

E) Hydroxyproline quantification of collagen in matrix deposited by tendon fibroblasts grown for $72 \mathrm{~h}$ with $10 \mu \mathrm{M}$ chloroquine or $1 \mathrm{nM}$ bafilomycin. $\mathrm{N}=3$ independent experiments for each treatment. Error bars represent SD.

\section{Extended data figure 8: Quantitative real time PCR of syntaxin-5 knock down.}

Quantitative real time PCR to detect Stx5 and Col1a1 transcripts in NIH3T3 cells treated with 100 pmol control scrambled (siScr) Stx5 targeting siRNA. Stx5 quantification is from $\mathrm{n}=3$ independent experiments each performed in triplicate. Error bars show SD. P value for Student's T-test is shown. Col1a1 quantification is based on the means of $\mathrm{N}=3$ independent experiments. Error bars show SEM. $P$ value for a paired Student's T-test is shown. 
727 Video 1: Intracellular collagen turnover over 2 days. A Dendra2::Col1a2 edited NIH3T3 cell was 728 photoswitched with $30 \mathrm{~s}$ exposure to $400 \mathrm{~nm}$ light. Cells were imaged every 20 minutes for 2 days. 729 The disappearance of photoswitched collagen occurs prior to visualization of new green Dendra2-PCI. 730 The video shows combined images captured using excitation and emission filters required to detect 731 GFP and mCherry.

732 Video 2: Deposition of Dendra2 tagged type I collagen into the matrix.

733 Time lapse microscopy of a Dendra2::Col1a2 edited NIH3T3 cell imaged $24 \mathrm{~h}$ after seeding. The cell 734 traced over a further $24 \mathrm{~h}$, with images taken every 20 minutes. Fibrils appear at approximately $36 \mathrm{~h}$ 735 after seeding and then grow rapidly.

736 Video 3: Dendra2 positive fibrils detected on the basal surface of cells.

737 Dendra2::Col1a2 NIH3T3 cell imaged after $48 \mathrm{~h}$ in culture. The video shows a step through of $51 \mathrm{Z}$ 738 planes starting from the basal surface. The nucleus of the cell is observed by the lack of Dendra 2 signal 739 within the cell body.

740 Video 4: Photoswitched Dendra2 positive fibrils detected on the basal surface of cells.

741 A 3D reconstruction of images of Dendra2::Col1a2 NIH3T3 cells transfected with BFP-KDEL after 742 photoswitching. Dendra2 positive fibrils (red/green) are formed on the basal surface of cells.

743 Video 5: Photoswitched Dendra2 positive fibrils detected on the basal surface of cells.

744 A 3D reconstruction of images of Dendra2::Col1a2 NIH3T3 cells transfected with BFP-KDEL after 745 photoswitching. Looped structures in Dendra2 positive fibrils (red/green) are engulfed by the cell with 746 the endoplasmic reticulum (BFP-KDEL) arranged around the looped fibril structure.

747 Video 6: Fibripositors are regularly contacted by endoplasmic reticulum and electron dense vesicles.

748 3view electron micrograph taken through embryonic mouse tendon showing a single fibripositor is 749 regularly contacted by both endoplasmic reticulum and electron dense vesicles.

750 Video 7: The Golgi apparatus does not contact fibripositors.

751 3view electron micrograph taken through embryonic mouse tendon showing that the Golgi apparatus

752 does not contact fibripositors.

753 TABLES

754 Table 1: Proteins identified in lysosomal fractions.

755 Table 2: Primers used for generating repair templates and BFP tagged HSP47.

756 Table 3: Primer sequences used to create split nanoluciferase knock-in repair templates.

757 Table 4: Primers used for real time PCR quantification of transcripts. 
Immortalized mouse embryonic fibroblasts, NIH3T3, were maintained in DMEM supplemented with $10 \%$ newborn bovine calf serum and penicillin and streptomycin. The preosteoblast cell line MC3T3 were maintained in alpha modification MEM supplemented with $10 \%$ fetal bovine serum and penicillin and streptomycin. Mouse tendon fibroblasts were cultured in DMEM:F-12 (1:1) medium supplemented with $5 \%$ L-glutamine, $10 \%$ dialyzed fetal bovine serum (FBS) and 10,000 U ml-1 penicillin/streptomycin) and at $37^{\circ} \mathrm{C}$ with $5 \% \mathrm{CO}$. For imaging, cells were grown on ibitreat $\mu$-Dish (Ibidi, Germany). Where indicated NIH3T3 and MC3T3 cell lines were cultured with 20 and $200 \mu \mathrm{g} / \mathrm{mL}$ L-ascorbic acid, respectively, to induce collagen fibril formation. Immortalized tail tendon fibroblasts were generated as previously described (Chang et al., 2020; Pickard et al., 2019) and were maintained in DMEM/F12 1:1 mixture supplemented with 10\% FBS, penicillin and streptomycin. For treatments, the following concentrations were used: bafilomycin A $100 \mathrm{nM}$, brefeldin A 100nM, monensin $1 \mu \mathrm{M}$, Chloroquine $1 \mu \mathrm{M}$ and ionomycin $10 \mu \mathrm{M}$. Cell survival was performed using Prestoblue blue (Thermo Fisher) after $72 \mathrm{~h}$ treatment, fluorescence measurements were collected on the Synergy Neo2 MultiMode Reader (Biotek) at Excitation: 555/20 nm, Emission: 596/20 nm (Xenon flash, Lamp energy low, Gain 100 and read height $4.5 \mathrm{~mm}, 10$ measurements per data point). For siRNA treatments, 200,000 cells were seeded in 6 well plates overnight and then transfected with 100 pmol siRNA, using RNAiMAX, protein, RNA and coverslips were harvested. Plasmid transfections were performed using Fugene 6 using $1 \mu \mathrm{g}$ plasmid and 3:1 (Fugene 6:DNA) ratio. BFP-KDEL was a gift from Gia Voeltz (Addgene plasmid \# 49150) (Friedman et al., 2011). HP47-BFP-RDEL was generated using PCR products of HP47 (PCR amplified from plasmid MC202692 (Origene)), and BFP-RDEL (PCR amplified from BFPKDEL plasmid) using primers detailed In Supplementary table 2. These were then assembled Into a pLenti CMV V5-LUC Blast (w567-1) digested with BstXI (a gift from Eric Campeau (Addgene plasmid \# 21474). Cy3 labeled collagen was prepared as previously described (Doyle, 2018). Cy3 collagen was added directly to medium at the indicated concentrations and mixed immediately.

\section{CRISPR/Cas9 knock-in design and vector construction}

786

We used CRISPR/Cas9 to generate the Col1A2-Dendra2 knock in cell line. Using the Sanger CRISPR design webtool we selected a guide RNA (ACTTACATTGGCATGTTGCT $\underline{\mathrm{AGG}}$ ) targeting exon 1 of the genomic sequence in order to generate a double strand break immediately after the sequence encoding the signal peptide. The guide was delivered as RNA oligos (integrated DNA technologies, Coralville, US) in complex with Cas9 protein. A double stranded DNA repair template was generated by PCR amplification of the $5^{\prime}$ and $3^{\prime}$ homology arms (800bp each) from mouse genomic DNA. The Dendra2 mouse optimized coding sequence with a flexible linker sequence was synthesized (Genscript, US) and assembled by Gibson Assembly (NEB), primers are shown in Supplementary table 2. Knockin of nanoluciferase to Col1a2 was previously described (Calverley et al., 2020).

795 To knock-in split nanoluciferase (Hibit) into mouse Col1a2 exon 6 we used gRNA: 796 GCTGCTCAGTATTCTGACAA. For human COL1A2 exon 1 knockin of Hibit we used gRNA: 797 CAAGCTGAAGGCACTTACAT. Repair templates for CRISPR-mediated knock-in of Hibit are detailed in 798 Supplementary table 2. Briefly the two primer sequences were annealed to generate hybrid 799 ssDNA:dsDNA repair templates with 29 bp homology arms. 
801

802

803

804

805

806

807

808

809

810

811

812

813

814

815

816

817

818

819

820

821

822

823

824

825

826

827

828

829

830

831

832

833

834

835

836

837

838

839

840

841

842

843

For knock-in of Dendra2, NIH3T3 and MC3T3 cells were seeded $24 \mathrm{~h}$ prior to transfection of the repair template, using Fugene 6 (Promega) at a ratio of $3 \mu \mathrm{L}$ per $1 \mu \mathrm{g}$ DNA, cells were maintained in transfection mixture overnight and then fresh medium was added for approximately $6 \mathrm{~h}$. Cells were then transfected overnight with 100 pmol of tracrRNA and crRNA complexed with 4 pmol recombinant cas9 protein using RNAiMAX (Thermo Fisher). Medium was then replaced and cells were grown for $48 \mathrm{~h}$ before assessing fluorescence and sorting.

For Hibit knockin crRNA were purchased from IDT, sequences are shown in Supplementary table 3. $0.25 \mu \mathrm{L}$ crRNA $(100 \mu \mathrm{M})$ was annealed with equimolar amounts tracRNA (IDT), and complexed with Cas9 (1.5 $\mu \mathrm{L} 1 \mu \mathrm{M}, \mathrm{NEB})$. CRISPR/Cas9 complexes and $100 \mathrm{pmol}$ annealed repair template were electroporated (Neon transfection system, Thermo). Electroporation conditions for NIH3T3 were 1400 $\mathrm{V}, 20 \mathrm{~ms}, 2$ pulses using $10 \mu \mathrm{L}$ tips and 50,000 cells and for HFFs were $1700 \mathrm{~V}, 30 \mathrm{~ms}$ and 1 pulse using $10 \mu \mathrm{L}$ tips and 100,000 cells. Electroporated cells were grown in 12 well plates.

\section{CRISPR/Cas9 validation}

Dendra2 positive populations were sorted by FACS and grown to sufficient numbers for validation of editing. Cells then sorted into single cells clones. All assays and imaging were performed on single cell sorted populations. Individual cells were then grown to sufficient numbers for validation. Primers for validation of the knockin into genomic DNA (PR1 and PR2) were as follows: FGGCAAGGGCGAGAGAGG and R-TTTTCTCCGACAGATTAGAGGGC. Real time validation of Dendra2::Col1a2 transcripts were assessed in single cell clones treated with $2.5 \mathrm{ng} / \mathrm{mL}$ TGF- $\beta 1$ for 48 $\mathrm{h}$ using the primers indicated in Supplementary table 4, real time PCR was normalized to the geometric mean of Rplp0, Gapdh and Actb. The sequence of the dendra2-col1a2 transcript was then validated by Sanger sequencing. Western blotting was performed on $4-12 \%$ tris-glycine gels with 25 $\mu \mathrm{g}$ cell lysate. Antibodies used in this study were Collagen (Gentaur, OARA02579, dilution 1:2000 (WB), 1:500 (IF)), Dendra2 (Origene, TA180094, dilution 1:500), GAPDH (Sigma, G8795, dilution 1:10,000) Calreticulin (Stressgen; SPA-601, dilution 1:1000), Lamp1 (Santa Cruz, sc-20011, 1:500), Vincullin (Chemicon; CBL233, 1:2000), Hp47 (Santa Cruz, sc-398579, 1:1000 (WB), 1:500 (IF)), Syntaxin 5 (Santa Cruz, sc-365124, 1:500(WB)) PDI (Abcam, ab180993, dilution 1:500 (IF)).

\section{Imaging}

For immunofluorescence detection of cellular and extracellular proteins, cells grown on coverslips were fixed with $4 \%$ paraformaldehyde for 20 minutes at room temperature and washed with PBS before permeabilization with $0.2 \%$ triton-x-100 in $10 \%$ FBS for 15 minutes. Antibodies used in this study are detailed above.

Figures $1 \mathrm{C}$ and $\mathrm{E}$, Video 1, Figures $\mathbf{5 A}$ and $\mathrm{E}$, and Extended data figure $\mathbf{5}$ were acquired on an Eclipse Ti inverted microscope (Nikon) using a 60x objective, the Nikon filter sets for GFP and mCherry and LED (Lumencor) fluorescent light sources each with $300 \mathrm{~ms}$ exposure. Photoswitching was performed using a 30s exposure to UV LED light source $(400 \mathrm{~nm}$ ). The images were collected using a Retiga R6 (QImaging) camera, and captured using NIS Elements AR.46.00.0 software. Pixel intensity was analyzed using FIJI ImageJ (http://imagej.net/Fiji/Downloads). Cells were maintained at $37^{\circ} \mathrm{C}$ and $5 \% \mathrm{CO}_{2}$.

Images from Extended data figure $\mathbf{2 A}$ and Figure 4C were collected on a Zeiss Axioimager.D2 upright microscope using a 100x objective and captured using a Coolsnap HQ2 camera (Photometrics) through Micromanager software v1.4.23. Following 4\% PFA fixation cells were permeabilized and stained with antibodies to Dendra2 (dilution 1:100) and collagen (dilution 1:200), secondary antibodies used were goat-anti-mouse488 (Cell Signaling; 4408, dilution 1:400) and goat anti-rabbit-Cy5 (Invitrogen; 
844 A10523, dilution 1:400). Specific band pass filter sets for FITC and Cy5 were used to prevent bleed

845 through from one channel to the next. All images were then processed and analyzed using Fiji.

846 Images for Figure $1 \mathrm{~F}$ and $\mathrm{G}$, Extended data figure 1G, Video 2, Figure $2 A$ and $D$, Extended data figure 847 2B, Video 3-5, Figure 4A, Figure 6A, Figure 7A and B, Extended data figure 6, Figure 8D, $E$ and $H$ and 848 Extended data figure 7B were acquired with either a Fluar 20X 0.75 NA objective at $1024 \times 1024$ 849 resolution, or 100x using a Zeiss LSM880 equipped with Airyscan detector set to super-resolution 850 mode. Green fluorescence was excited at $488 \mathrm{~nm}$ and collected through a $495-550 \mathrm{~nm}$ filter. The 32 851 phase images were recombined using the Airyscan processing tool in the Zeiss Zen 2 software and the 852 image brightness and contrast adjusted using BestFit. Extended data figure 2G and Figure 3G were 853 captured were acquired with either a Fluar 20X 0.75NA objective with Airyscan detector set to super854 resolution mode. Green fluorescence was excited at $488 \mathrm{~nm}$ and collected through a $495-550 \mathrm{~nm}$ filter.

855 The 32 phase images were recombined using the Airyscan processing tool in the Zeiss Zen 2 software 856 and the image brightness and contrast adjusted using BestFit.

857 Figures $6 C, 7 C, 9 A$ and B, and Extended data figure 6 were collected on a Leica TCS SP5 AOBS inverted 858 confocal using an [63x / 0.50 Plan Fluotar] objective. The confocal settings were as follows, pinhole [1 859 airy unit], scan speed [1000 Hz unidirectional]. Images were collected using [PMT] detectors with the 860 following detection mirror settings; [FITC $494-530 \mathrm{~nm}$; Cy5 640-690 nm] using the [488 nm (20\%) and $861633 \mathrm{~nm}(25 \%)$ ] laser lines respectively. To eliminate crosstalk between channels images were collected 862 sequentially.

\section{Electron microscopy}

864 Transmission electron microscopy: Dendra2::Col1a2 edited MC3T3 were grown for 7 days in the 865 presence of $200 \mu \mathrm{g} / \mathrm{mL}$ L-ascorbic acid, replenishing medium and ascorbic acid every 2 days. Following fixation in $2.5 \%$ glutaraldehyde/100 mM phosphate buffer $(\mathrm{pH} \mathrm{7.2)} \mathrm{for} 30$ minutes at RT cells and matrix were scraped from the culture dish. After $2 \mathrm{~h}$ at $4 \stackrel{\circ}{\circ} \mathrm{C}$ the samples were washed in $100 \mathrm{mM}$ phosphate buffer ( $\mathrm{pH}$ 7.2). After immersion in $2 \%$ osmium $/ 1.5 \%$ potassium ferro-cyanide in cacodylate buffer ( $\mathrm{pH}$ 7.2) for $1 \mathrm{~h}$ at RT, samples were washed in $\mathrm{ddH} 2 \mathrm{O}$, and fixed in $1 \%$ tannic acid/0.1 M cacodylate buffer $\left(\mathrm{pH} \mathrm{7.2)}\right.$ for $2 \mathrm{~h}$ at $4{ }^{\circ} \mathrm{C}$. Samples were then thoroughly washed in $\mathrm{ddH} 2 \mathrm{O}$ and incubated in $2 \%$ osmium tetroxide/ddH2O for $40 \mathrm{~min}$ at RT, before washing again in ddH2O at RT. This was followed by a final incubation step at $4 \stackrel{\circ}{\circ}$ in $1 \%$ uranyl acetate (aqueous) overnight. Samples were then washed before infiltrated with a series of propylene oxide and TAAB 812 resin kit mix, with increasing resin concentration ( $2 \mathrm{~h}$ in 30\% resin, $2 \mathrm{~h}$ in 50\% resin, $2 \mathrm{~h}$ in 75\% resin, $3 \times 1 \mathrm{~h}$ in 100\% resin). Samples are then embedded in capsules and cured at $60^{\circ} \mathrm{C}$ for $12 \mathrm{~h}$. Sections ( $80 \mathrm{~nm}$ thick) were cut from the sample blocks and examined using a Tecnai 12 BioTwin electron microscope. Serial block face SEM: Tendons were prepared as described previously (Starborg et al., 2013). In brief, 878 tendons were fixed in $1 \%$ osmium and $1.5 \%$ potassium ferrocyanide in $0.1 \mathrm{M}$ sodium cacodylate buffer for $1 \mathrm{~h}$, washed with dd $\mathrm{H}_{2} \mathrm{O}$ water, then incubated with $1 \%$ tannic acid in $0.1 \mathrm{M}$ cacodylate buffer for $8801 \mathrm{~h}$, washed, then incubated with $1 \%$ osmium tetroxide in water for $30 \mathrm{~min}$. Samples were then washed with $\mathrm{ddH}_{2} \mathrm{O}$ water and stained with $1 \%$ uranyl acetate in water for $1 \mathrm{~h}$, then dehydrated in acetone and embedded into resin. anti-rabbit antibody (British Biocell International) at a dilution of 1:200. All sections were 

microscope.

\section{Atomic force microscopy}

889 Atomic force microscopy was performed using a JPK NanoWizard IV (Bruker Nano Inc., Karlsruhle, 890 Germany, previously JPK Instruments AG, Berlin, Germany) mounted on a Zeiss AX10 (Carl Zeiss 891 Microscopy $\mathrm{GmbH}$, Jena, Germany) inverted light microscope operating under JPK NanoWizard 892 Control software (V 6.1.65). Images were captured using NuSense Scout 350R cantilevers (NuNano, 893 Bristol, UK) with nominal spring constant, frequency and tip radius of $42 \mathrm{~N} / \mathrm{m}, 350 \mathrm{kHz}$ and $<10 \mathrm{~nm}$ 894 respectively. Height data was processed using JPK Data Processing software (V 6.1.65), and was $1^{\text {st }}$ 895 order flattened prior to analysis.

\section{Nanoluciferase activity assay}

897 Conditioned medium collected after washing edited cells twice with PBS, was placed in white walled 89896 well plates (Nunc MicroWell 96-Well, Nunclon Delta-Treated, Flat-Bottom Microplate, Thermo 899 Fisher Scientific, Paisley, UK\# 136101) To assay Nluc activity $0.5 \mu \mathrm{L}$ of coelenterazine (final 900 concentration $3 \mu \mathrm{M}$ ) was added immediately prior to measurement. Light production was measured 901 using filter cubes \#114 and \#3 on the Synergy Neo2 Multi-Mode Reader (Biotek), readings for each 902 well were integrated over $200 \mathrm{~ms}$ with 4 replicate measurements per well (Gain 135 and read height $9036 \mathrm{~mm}$ ). For assessment of cellular and matrix derived Nluc activity cultures were decellularized by 904 addition of extraction buffer ( $20 \mathrm{mM} \mathrm{NH}_{4} \mathrm{OH}, 0.5 \%$ Triton X-100 in PBS) for 2 mins at 37 으 until no 905 intact cell is visible under light microscope, this cellular fraction was removed and the matrix was 906 washed with PBS, and then scraped into $1 \mathrm{~mL}$ PBS and pelleted by centrifugation at 12,000 $\mathrm{g}$ for 5 907 mins. Matrix was resuspended in $100 \mu \mathrm{L}$ extraction buffer before assessing Nluc activity.

\section{Hydroxyproline Assay}

909 Decellularized cultures were scraped and pelleted into $1.5 \mathrm{~mL}$ tubes before freezing at $-20{ }^{\circ} \mathrm{C}$ for the 910 hydroxyproline quantitation. Hydroxyproline was measured using methods previously described 911 (Reddy and Enwemeka, 1996). Briefly, $100 \mu \mathrm{L} 6 \mathrm{M} \mathrm{HCl}$ was added to the pellet and incubated at 100 $912{ }^{\circ} \mathrm{C}$ overnight. Samples were cooled to room temperature and spun at $12,000 \mathrm{xg}$ for 3 mins to remove 913 residual charcoal. For each sample $(50 \mu \mathrm{L})$ was mixed with chloramine $\mathrm{T}(450 \mu \mathrm{L})$ and incubated at 914 room temperature for 25 mins. Ehrlich's reagent $(500 \mu \mathrm{L})$ was added to each sample and incubated at $91565{ }^{\circ} \mathrm{C}$ for $10 \mathrm{mins}$. All samples were compared to hydroxyproline standards treated identically. The 916 absorbance of $100 \mu \mathrm{L}$ was measured a 96-well plate and absorbance at $558 \mathrm{~nm}$ read on a $\mathrm{H} 1$ plate 917 reader (Biotek).

\section{Lysosomal fractionation}

91930 million cells were grown in $150 \mathrm{~mm}$ tissue culture dishes, trypsinized and pelleted at $1000 \mathrm{xg}$ for 5 920 minutes. Lysosomal fractions were prepared using the Lysosome Isolation Kit (Sigma Aldrich, LYSISO1) 921 according to manufacturer's procedures. 9 fractions were collected after ultracentrifugation.

\section{Proteomic sample preparation}

923 Cell pellets were resuspended in $30 \mu \mathrm{LL}-\mathrm{DOC}$ (1.1\% sodium laurate, $0.3 \%$ sodium deoxycholate in 25 $924 \mathrm{mM}$ ammonium bicarbonate supplemented with protease and phosphatase inhibitor cocktails). Six $9251.6 \mathrm{~mm}$ steel beads were added and the samples were homogenized in a Bullet Blender Tissue 926 Homogeniser. A BCA was done to quantify the amount of protein in each sample. Each sample (50 $927 \mu \mathrm{g}$ ) was made up to 5\% SDS and then reduced and alkylated with DTT and lodoacetamide (IAA) 928 respectively. For lysosome fractions, samples were processed without further extraction procedures. 

was added. Samples were loaded, onto S-Trap columns (ProtiFi) and washed with S-trap binding buffer 4 times. Proteins were digested with $0.8 \mu \mathrm{g} / \mu \mathrm{L}$ trypsin solution (proteomics grade trypsin, Promega). Peptides were then eluted in $65 \mu$ l Digestion buffer (50 mM TEAB pH 8.5), $65 \mu \mathrm{L} 0.1 \%$ formic acid (in water) and finally with $30 \mu \mathrm{L} 0.1 \%$ formic acid, $30 \%$ Acetonitrile (ACN) (in water). Samples were then desalted using Oligo R3 resin beads in a 96-well, $0.2 \mu \mathrm{m}$ PVDF filter plate (Corning). The beads were washed and then samples added and washed twice with $0.1 \%$ formic acid. Samples were then eluted in $0.1 \%$ formic acid in $30 \%$ ACN and lyophilized using a speed-vac (Heto Cooling System).

\section{Mass Spectrometry}

938 Dried peptides were resuspended in $10 \mu \mathrm{l} 0.1 \%$ formic acid in 5\% ACN. Samples were analyzed using 939 an ultiMate ${ }^{\circledR} 3000$ Rapid Separation LC system (RSLC, Dionex Corporation) coupled to first a Orbitrap 940 Elite, for quality control, and then a Q Exactive HF Mass Spectrometer (Thermo Fisher). For both, 941 mobile phase A was $0.1 \%$ formic acid in water and B was $0.1 \%$ formic acid in ACN. The Orbitrap used 942 a $75 \mathrm{~mm} \times 250$ um inner diameter $1.7 \mu \mathrm{M}$ CSH C18 analytical column (Waters) with a gradient from $94392 \% A$ and $8 \% B$ to $33 \% B$ in 10 minutes at a rate of $300 \mathrm{~nL} / \mathrm{min}$. The $Q$ Exactive used a gradient of $95 \%$ $944 \mathrm{~A}$ and $5 \% \mathrm{~B}$ to $18 \% \mathrm{~B}$ at 58 minutes, $27 \%$ at $72 \mathrm{~min}$ and $60 \%$ at $74 \mathrm{~min}$ with the same flow rate and a $94575 \mathrm{~mm} \times 250 \mu \mathrm{m}$ inner diameter CSH C18 analytical column (Waters). Peptides were selected by DDA for fragmentation automatically and data was acquired for $90 \mathrm{~min}$ in positive mode.

\section{SILAC labelling}

948 Mouse tendon fibroblasts were pulsed with $100 \mathrm{mg} / \mathrm{L} \mathrm{C}^{13}$ ("Heavy") lysine for $48 \mathrm{~h}$ and then washed, 949 trypsinized, lysed and processed for mass spectrometry (see below). Mass spectrometry results files 950 were exported into Proteome Discoverer for identification and quantification using a SILAC 1plex 951 (Lys6) method. All searches included the fixed modification for carbamidomethylation on cysteine 952 residues resulting from IAA treatment to prevent cysteine bonding. The variable modifications included in the search were oxidized methionine (monoisotopic mass change, $+15.955 \mathrm{Da}$ ) and phosphorylation of threonine, serine and tyrosine (79.966 Da). A maximum of 2 missed cleavages per peptide was allowed. The minimum precursor mass was set to 350 Da with a maximum of $5000 \mathrm{Da}$. Precursor mass tolerance was set to $10 \mathrm{ppm}$, fragment mass tolerance was $0.02 \mathrm{Da}$ and minimum peptide length was 6. Peptides were searched against the Swissprot database using Sequest HT with a maximum false discovery rate of $1 \%$.

959 Half-lives were calculated from heavy to light ratios (HL) as shown by Schwanhäusser using the 960 following equations (Schwanhausser et al., 2009):

$$
k=\frac{\ln (H L+1)}{48}-\frac{\ln (2)}{72} \quad t_{1 / 2}=\frac{\ln (2)}{k}
$$

961 Where 48 represents the SILAC pulse time, 72 is the calculated cell doubling rate and $\mathrm{k}$ is the rate constant of protein decay, which is then used to calculate the half-life in the second equation.

This calculation assumes that no new light protein is produced and that the amount of light protein decays exponentially over time. The total amount of protein is assumed to double per complete cell cycle.

966 Proteins were only selected for half-life calculation if at least 3 peptides were detected, where at least 
970 Beck, G.R., Jr., Zerler, B., and Moran, E. (2001). Gene array analysis of osteoblast differentiation. Cell Growth

971 Differ 12, 61-83.

972 Birk, D.E., and Trelstad, R.L. (1984). Extracellular compartments in matrix morphogenesis: collagen fibril, 973 bundle, and lamellar formation by corneal fibroblasts. J Cell Biol 99, 2024-2033. 10.1083/jcb.99.6.2024.

974 Birk, D.E., and Trelstad, R.L. (1986). Extracellular compartments in tendon morphogenesis: collagen fibril, 975 bundle, and macroaggregate formation. J Cell Biol 103, 231-240. 10.1083/jcb.103.1.231.

976 Blott, E.J., and Griffiths, G.M. (2002). Secretory lysosomes. Nat Rev Mol Cell Biol 3, 122-131. 10.1038/nrm732.

977 Bonfanti, L., Mironov, A.A., Jr., Martinez-Menarguez, J.A., Martella, O., Fusella, A., Baldassarre, M., Buccione,

978 R., Geuze, H.J., Mironov, A.A., and Luini, A. (1998). Procollagen traverses the Golgi stack without leaving the

979 lumen of cisternae: evidence for cisternal maturation. Cell 95, 993-1003. 10.1016/s0092-8674(00)81723-7.

980 Bonifacino, J.S., and Hurley, J.H. (2008). Retromer. Curr Opin Cell Biol 20, 427-436. 10.1016/j.ceb.2008.03.009.

981 Buratta, S., Tancini, B., Sagini, K., Delo, F., Chiaradia, E., Urbanelli, L., and Emiliani, C. (2020). Lysosomal

982 Exocytosis, Exosome Release and Secretory Autophagy: The Autophagic- and Endo-Lysosomal Systems Go

983 Extracellular. Int J Mol Sci 21. 10.3390/ijms21072576.

984 Calverley, B.C., Kadler, K.E., and Pickard, A. (2020). Dynamic High-Sensitivity Quantitation of Procollagen-I by

985 Endogenous CRISPR-Cas9 NanoLuciferase Tagging. Cells 9. 10.3390/cells9092070.

986 Canty, E.G., and Kadler, K.E. (2005). Procollagen trafficking, processing and fibrillogenesis. J Cell Sci 118, 1341-

987 1353. 10.1242/jcs.01731.

988 Canty, E.G., Lu, Y., Meadows, R.S., Shaw, M.K., Holmes, D.F., and Kadler, K.E. (2004). Coalignment of plasma 989 membrane channels and protrusions (fibripositors) specifies the parallelism of tendon. J Cell Biol 165, 553-563. $99010.1083 / j c b .200312071$.

991 Chang, J., Garva, R., Pickard, A., Yeung, C.C., Mallikarjun, V., Swift, J., Holmes, D.F., Calverley, B., Lu, Y., 992 Adamson, A., et al. (2020). Circadian control of the secretory pathway maintains collagen homeostasis. Nat 993 Cell Biol 22, 74-86. 10.1038/s41556-019-0441-z.

994 Colanzi, A., Grimaldi, G., Catara, G., Valente, C., Cericola, C., Liberali, P., Ronci, M., Lalioti, V.S., Bruno, A., 995 Beccari, A.R., et al. (2013). Molecular mechanism and functional role of brefeldin A-mediated ADP-ribosylation 996 of CtBP1/BARS. Proc Natl Acad Sci U S A 110, 9794-9799. 10.1073/pnas.1222413110.

997 Craig, A.S., Birtles, M.J., Conway, J.F., and Parry, D.A. (1989). An estimate of the mean length of collagen fibrils 998 in rat tail-tendon as a function of age. Connect Tissue Res 19, 51-62. 10.3109/03008208909016814. 
Doan, N.-D., DiChiara, A.S., Del Rosario, A.M., Schiavoni, R.P., and Shoulders, M.D. (2019). Mass spectrometry-

1000 based proteomics to define intracellular collagen interactomes. In Collagen. Methods in Molecular Biology, A.

1001 Sagi l., N., ed. (Humana Press, New York, NY), pp. 95-114. http://doi.org/10.1007/978-1-4939-9095-5 7.

1002 Doyle, A.D. (2018). Fluorescent Labeling of Rat-tail Collagen for 3D Fluorescence Imaging. Bio Protoc 8.

1003 10.21769/BioProtoc.2919.

1004 Fedele, A.O., and Proud, C.G. (2020). Chloroquine and bafilomycin A mimic lysosomal storage disorders and

1005 impair mTORC1 signalling. Biosci Rep 40. 10.1042/BSR20200905.

1006 Forrester, A., De Leonibus, C., Grumati, P., Fasana, E., Piemontese, M., Staiano, L., Fregno, I., Raimondi, A.,

1007 Marazza, A., Bruno, G., et al. (2019). A selective ER-phagy exerts procollagen quality control via a Calnexin-

1008 FAM134B complex. EMBO J 38. 10.15252/embj.201899847.

1009 Friedman, J.R., Lackner, L.L., West, M., DiBenedetto, J.R., Nunnari, J., and Voeltz, G.K. (2011). ER tubules mark

1010 sites of mitochondrial division. Science 334, 358-362. 10.1126/science.1207385.

1011 Gorrell, L., Omari, S., Makareeva, E., and Leikin, S. (2021). Noncanonical ER-Golgi trafficking and autophagy of 1012 endogenous procollagen in osteoblasts. Cell Mol Life Sci 78, 8283-8300. 10.1007/s00018-021-04017-z.

1013 Graham, H.K., Holmes, D.F., Watson, R.B., and Kadler, K.E. (2000). Identification of collagen fibril fusion during

1014 vertebrate tendon morphogenesis. The process relies on unipolar fibrils and is regulated by collagen-

1015 proteoglycan interaction. J Mol Biol 295, 891-902. 10.1006/jmbi.1999.3384.

1016 Harris, J.R., and Reiber, A. (2007). Influence of saline and pH on collagen type I fibrillogenesis in vitro: fibril

1017 polymorphism and colloidal gold labelling. Micron 38, 513-521. 10.1016/j.micron.2006.07.026.

1018 Heinemeier, K.M., Schjerling, P., Heinemeier, J., Magnusson, S.P., and Kjaer, M. (2013). Lack of tissue renewal 1019 in human adult Achilles tendon is revealed by nuclear bomb (14)C. FASEB J 27, 2074-2079. 10.1096/fj.121020225599.

1021 Heinemeier, K.M., Schjerling, P., Heinemeier, J., Moller, M.B., Krogsgaard, M.R., Grum-Schwensen, T., 1022 Petersen, M.M., and Kjaer, M. (2016). Radiocarbon dating reveals minimal collagen turnover in both healthy 1023 and osteoarthritic human cartilage. Sci Transl Med 8, 346ra390. 10.1126/scitransImed.aad8335.

1024 Hodgson, M.J., Knutson, C.C., Momtahan, N., and Cook, A.D. (2018). Extracellular Matrix from Whole Porcine 1025 Heart Decellularization for Cardiac Tissue Engineering. Methods Mol Biol 1577, 95-102.

1026 10.1007/7651_2017_31.

1027 Holmes, D.F., Watson, R.B., Steinmann, B., and Kadler, K.E. (1993). Ehlers-Danlos syndrome type VIIB.

1028 Morphology of type I collagen fibrils formed in vivo and in vitro is determined by the conformation of the

1029 retained N-propeptide. J Biol Chem 268, 15758-15765. 
1030 Hulmes, D.J., Kadler, K.E., Mould, A.P., Hojima, Y., Holmes, D.F., Cummings, C., Chapman, J.A., and Prockop,

1031 D.J. (1989). Pleomorphism in type I collagen fibrils produced by persistence of the procollagen N-propeptide. J

1032 Mol Biol 210, 337-345. 10.1016/0022-2836(89)90335-5.

1033 Humphries, S.M., Lu, Y., Canty, E.G., and Kadler, K.E. (2008). Active negative control of collagen fibrillogenesis 1034 in vivo. Intracellular cleavage of the type I procollagen propeptides in tendon fibroblasts without intracellular 1035 fibrils. J Biol Chem 283, 12129-12135. 10.1074/jbc.M708198200.

1036 Ishikawa, Y., and Bachinger, H.P. (2013). A molecular ensemble in the rER for procollagen maturation. Biochim 1037 Biophys Acta 1833, 2479-2491. 10.1016/j.bbamcr.2013.04.008.

1038 Jackson, D.S., and Fessler, J.H. (1955). Isolation and properties of a collagen soluble in salt solution at neutral $1039 \mathrm{pH}$. Nature 176, 69-70. 10.1038/176069a0.

1040 Kadler, K.E., Hojima, Y., and Prockop, D.J. (1987). Assembly of collagen fibrils de novo by cleavage of the type I 1041 pC-collagen with procollagen C-proteinase. Assay of critical concentration demonstrates that collagen self1042 assembly is a classical example of an entropy-driven process. J Biol Chem 262, 15696-15701.

1043 Kadler, K.E., Hojima, Y., and Prockop, D.J. (1988). Assembly of type I collagen fibrils de novo. Between 37 and 104441 degrees $C$ the process is limited by micro-unfolding of monomers. J Biol Chem 263, 10517-10523.

1045 Kadler, K.E., Hojima, Y., and Prockop, D.J. (1990). Collagen fibrils in vitro grow from pointed tips in the C- to N1046 terminal direction. Biochem J 268, 339-343. 10.1042/bj2680339.

1047 Kalson, N.S., Starborg, T., Lu, Y., Mironov, A., Humphries, S.M., Holmes, D.F., and Kadler, K.E. (2013).

1048 Nonmuscle myosin II powered transport of newly formed collagen fibrils at the plasma membrane. Proc Natl

1049 Acad Sci U S A 110, E4743-4752. 10.1073/pnas.1314348110.

1050 Kapacee, Z., Richardson, S.H., Lu, Y., Starborg, T., Holmes, D.F., Baar, K., and Kadler, K.E. (2008). Tension is 1051 required for fibripositor formation. Matrix Biol 27, 371-375. 10.1016/j.matbio.2007.11.006.

1052 Kim, J., Gee, H.Y., and Lee, M.G. (2018). Unconventional protein secretion - new insights into the pathogenesis 1053 and therapeutic targets of human diseases. J Cell Sci 131. 10.1242/jcs.213686.

1054 Kubow, K.E., Vukmirovic, R., Zhe, L., Klotzsch, E., Smith, M.L., Gourdon, D., Luna, S., and Vogel, V. (2015).

1055 Mechanical forces regulate the interactions of fibronectin and collagen I in extracellular matrix. Nat Commun 1056 6, 8026. 10.1038/ncomms9026.

1057 Lamande, S.R., and Bateman, J.F. (1999). Procollagen folding and assembly: the role of endoplasmic reticulum 1058 enzymes and molecular chaperones. Semin Cell Dev Biol 10, 455-464. 10.1006/scdb.1999.0317. 
Li, I.M.H., Horwell, A.L., Chu, G., de Crombrugghe, B., and Bou-Gharios, G. (2017). Characterization of Mesenchymal-Fibroblast Cells Using the Col1a2 Promoter/Enhancer. Methods Mol Biol 1627, 139-161. 10.1007/978-1-4939-7113-8_10.

1064 McCaughey, J., Stevenson, N.L., Cross, S., and Stephens, D.J. (2019). ER-to-Golgi trafficking of procollagen in the absence of large carriers. J Cell Biol 218, 929-948. 10.1083/jcb.201806035. general role for TANGO1, encoded by MIA3, in secretory pathway organization and function. J Cell Sci 134. 10.1242/jcs.259075.

1069 Mirigian, L.S., Makareeva, E., and Leikin, S. (2014). Pulse-chase analysis of procollagen biosynthesis by azidohomoalanine labeling. Connect Tissue Res 55, 403-410. 10.3109/03008207.2014.959120. accommodates enhanced collagen production during differentiation. J Biol Chem 283, 19678-19690. 10.1074/jbc.M802517200. Malhotra, V. (2014). SLY1 and Syntaxin 18 specify a distinct pathway for procollagen VII export from the endoplasmic reticulum. Elife 3, e02784. 10.7554/eLife.02784.

1077 Parry, D.A., Barnes, G.R., and Craig, A.S. (1978). A comparison of the size distribution of collagen fibrils in

1078 connective tissues as a function of age and a possible relation between fibril size distribution and mechanical properties. Proc R Soc Lond B Biol Sci 203, 305-321. 10.1098/rspb.1978.0107.

1080 Parry, D.A., and Craig, A.S. (1977). Quantitative electron microscope observations of the collagen fibrils in rattail tendon. Biopolymers 16, 1015-1031. 10.1002/bip.1977.360160506. 10.1096/fj.201802366RR. Golgi apparatus and plasma membrane. J Cell Biol 148, 45-58. 10.1083/jcb.148.1.45.

1088 Raote, I., and Malhotra, V. (2021). Tunnels for Protein Export from the Endoplasmic Reticulum. Annu Rev 
1090 Reddy, A., Caler, E.V., and Andrews, N.W. (2001). Plasma membrane repair is mediated by Ca(2+)-regulated

1091 exocytosis of lysosomes. Cell 106, 157-169. 10.1016/s0092-8674(01)00421-4.

1092 Reddy, G.K., and Enwemeka, C.S. (1996). A simplified method for the analysis of hydroxyproline in biological tissues. Clin Biochem 29, 225-229. 10.1016/0009-9120(96)00003-6.

1094 Ricard-Blum, S. (2011). The collagen family. Cold Spring Harb Perspect Biol 3, a004978.

1095 10.1101/cshperspect.a004978.

1096 Rodriguez, A., Webster, P., Ortego, J., and Andrews, N.W. (1997). Lysosomes behave as Ca2+-regulated 1097 exocytic vesicles in fibroblasts and epithelial cells. J Cell Biol 137, 93-104. 10.1083/jcb.137.1.93.

1098 Schwanhausser, B., Gossen, M., Dittmar, G., and Selbach, M. (2009). Global analysis of cellular protein 1099 translation by pulsed SILAC. Proteomics 9, 205-209. 10.1002/pmic.200800275.

1100 Smejkal, G.B., and Fitzgerald, C. (2017). Revised estimate of total collagen in the human body. Journal of 1101 Proteome Bioinformatics 2, 001-002.

1102 Song, J.J., Guyette, J.P., Gilpin, S.E., Gonzalez, G., Vacanti, J.P., and Ott, H.C. (2013). Regeneration and 1103 experimental orthotopic transplantation of a bioengineered kidney. Nat Med 19, 646-651. 10.1038/nm.3154.

1104 Starborg, T., Kalson, N.S., Lu, Y., Mironov, A., Cootes, T.F., Holmes, D.F., and Kadler, K.E. (2013). Using 1105 transmission electron microscopy and 3View to determine collagen fibril size and three-dimensional 1106 organization. Nat Protoc 8, 1433-1448. 10.1038/nprot.2013.086.

1107 Stenseth, K., and Thyberg, J. (1989). Monensin and chloroquine inhibit transfer to lysosomes of endocytosed 1108 macromolecules in cultured mouse peritoneal macrophages. Eur J Cell Biol 49, 326-333.

1109 Stevenson, N.L., Bergen, D.J.M., Lu, Y., Prada-Sanchez, M.E., Kadler, K.E., Hammond, C.L., and Stephens, D.J. 1110 (2021). Giantin is required for intracellular N-terminal processing of type I procollagen. J Cell Biol 220.

$1111 \quad 10.1083 /$ jcb.202005166.

1112 Trelstad, R.L., and Hayashi, K. (1979). Tendon collagen fibrillogenesis: intracellular subassemblies and cell 1113 surface changes associated with fibril growth. Dev Biol 71, 228-242. 10.1016/0012-1606(79)90166-0.

1114 Trotter, J.A., Kadler, K.E., and Holmes, D.F. (2000). Echinoderm collagen fibrils grow by surface-nucleation-and1115 propagation from both centers and ends. J Mol Biol 300, 531-540. 10.1006/jmbi.2000.3879.

1116 Ushiki, T. (2002). Collagen fibers, reticular fibers and elastic fibers. A comprehensive understanding from a 1117 morphological viewpoint. Arch Histol Cytol 65, 109-126. 10.1679/aohc.65.109. 
1118 Vaben, C., Heinemeier, K.M., Schjerling, P., Olsen, J., Petersen, M.M., Kjaer, M., and Krogsgaard, M.R. (2020).

1119 No detectable remodelling in adult human menisci: an analysis based on the $\mathrm{C}(14)$ bomb pulse. Br J Sports

1120 Med 54, 1433-1437. 10.1136/bjsports-2019-101360.

1121 van der Sluijs, P., Zibouche, M., and van Kerkhof, P. (2013). Late steps in secretory lysosome exocytosis in

1122 cytotoxic lymphocytes. Front Immunol 4, 359. 10.3389/fimmu.2013.00359.

1123 Wang, H., Han, X., and Xu, J. (2020). Lysosome as the Black Hole for Checkpoint Molecules. Adv Exp Med Biol 1124 1248, 325-346. 10.1007/978-981-15-3266-5_14.

1125 Wickstrom, S.A., and Fassler, R. (2011). Regulation of membrane traffic by integrin signaling. Trends Cell Biol 1126 21, 266-273. 10.1016/j.tcb.2011.02.003.

1127 Yang, J., Wheeler, S.E., Velikoff, M., Kleaveland, K.R., LaFemina, M.J., Frank, J.A., Chapman, H.A., Christensen, 1128 P.J., and Kim, K.K. (2013). Activated alveolar epithelial cells initiate fibrosis through secretion of mesenchymal 1129 proteins. Am J Pathol 183, 1559-1570. 10.1016/j.ajpath.2013.07.016.

1130 Young, R.D., Knupp, C., Pinali, C., Png, K.M., Ralphs, J.R., Bushby, A.J., Starborg, T., Kadler, K.E., and Quantock, 1131 A.J. (2014). Three-dimensional aspects of matrix assembly by cells in the developing cornea. Proc Natl Acad Sci 1132 U S A 111, 687-692. 10.1073/pnas.1313561110.

1133 


\section{Figures}
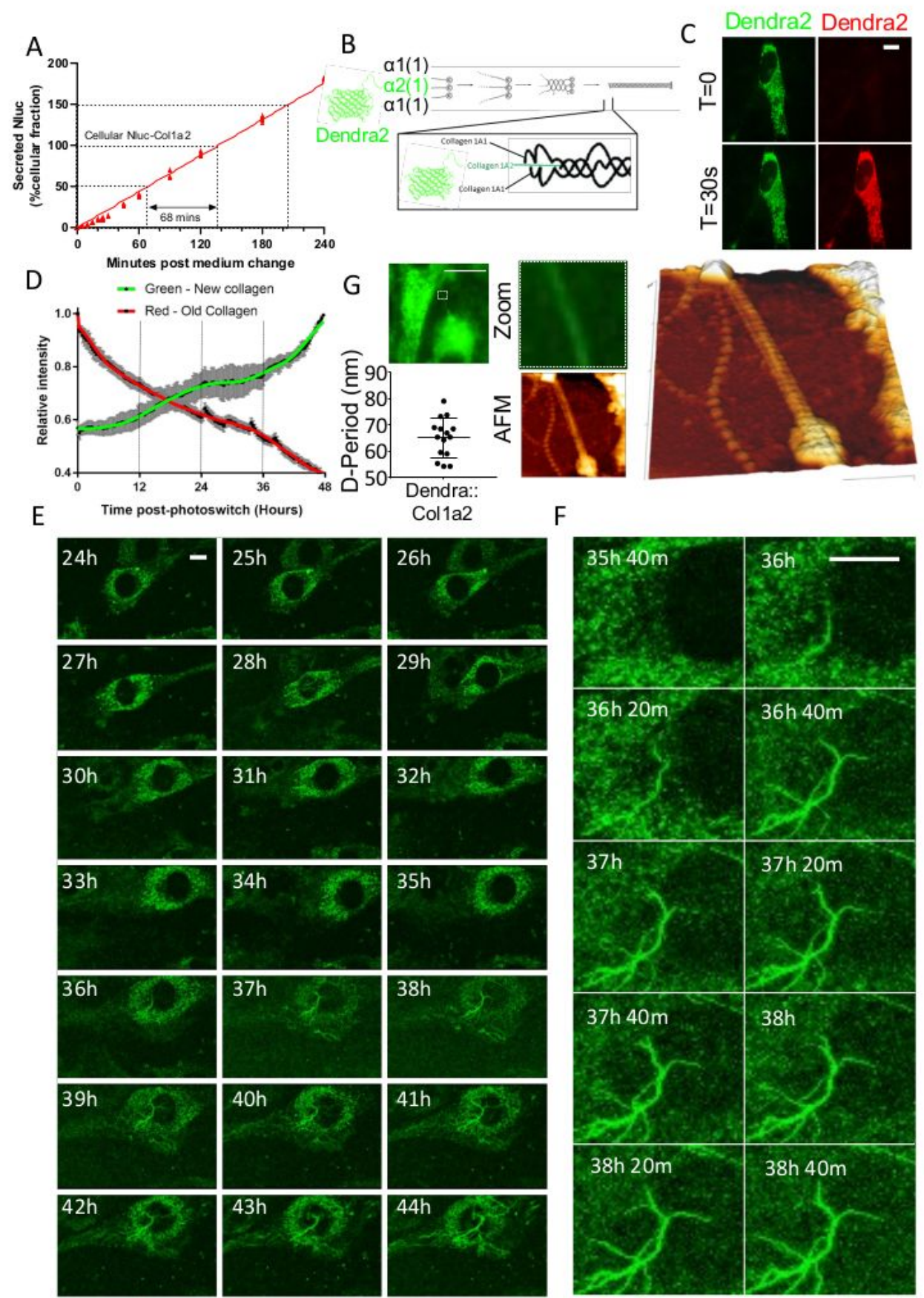

\section{Figure 1}

$\mathrm{PCl}$ secretion and collagen fibril assembly are separate processes. A) Assessment of collagen secretion rate in CRISPR-edited Nluc::Col1a2 NIH3T3 cells. Nluc activity is readily detected in conditioned medium within minutes after medium change. Comparison to cellular Nluc::Col1a2 activity levels were used to 
assess the rate of type-I collagen secretion. $\mathrm{N}=4$, individual data points are shown. B) CRISPR strategy to assess the lifetime of type I collagen intracellularly. Integration of the photoswitchable Dendra2 into the $\mathrm{N}$-terminus of Col1a2, the short N-terminus of Col1a2 provides space within the N-pro-peptide for incorporation of Dendra2 into the type 1 collagen triple helix. Image modified from (Canty and Kadler, 2005). C) Photoswitching of Dendra2::Col1a2 after 30 second exposure to $405 \mathrm{~nm}$ light. D) Tracing of pre-existing Dendra2::Col1a2 (Red) pixel intensity demonstrating a slower rate of type-I collagen secretion, and a phased collagen production of new Dendra2::Col1a2, intensities are relative to the first frame after photoswitching (Red) or to the final frame (Green). The average pixel intensities of five cells are shown. E) Dendra2::Col1a2 NIH3T3 cells grown on correlative grid coverslips imaged by widefield fluorescence microscopy, samples were then fixed to perform AFM of the same region. Assessment of the periodicity of the Dendra2 positive fibril demonstrated a D-Period of $65 \mathrm{~nm} .11 \mathrm{~F}$ ) Time-lapse Airyscan microscopy tracing Dendra2::Col1a2 NIH3T3 cells, $24 \mathrm{~h}$ after seeding no fibrillar structures were detected but with prolonged culture Dendra2 positive fibrillar structures were detected. G) Zoom of the cell imaged in $\mathrm{F}$ at the position of fibril formation. Scale bars represent $10 \mu \mathrm{m}$. 


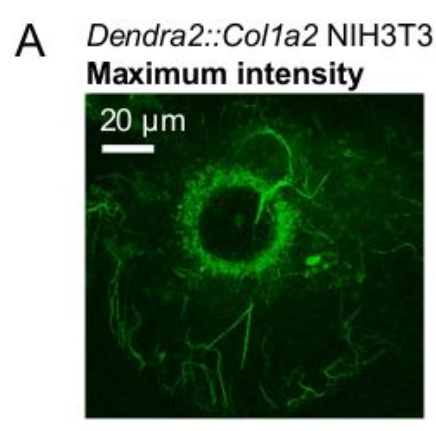

$\mathrm{B}$

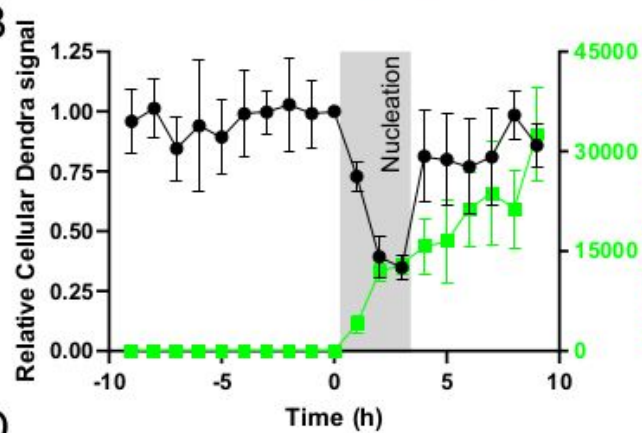

D

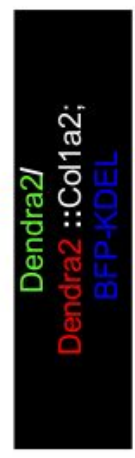

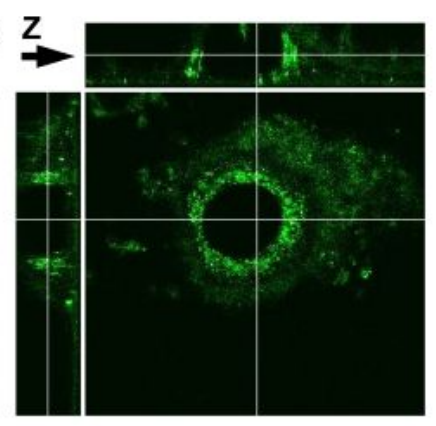

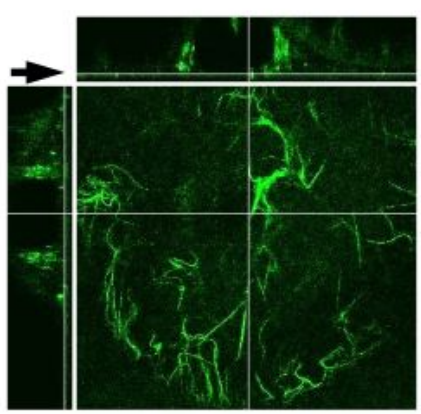

C

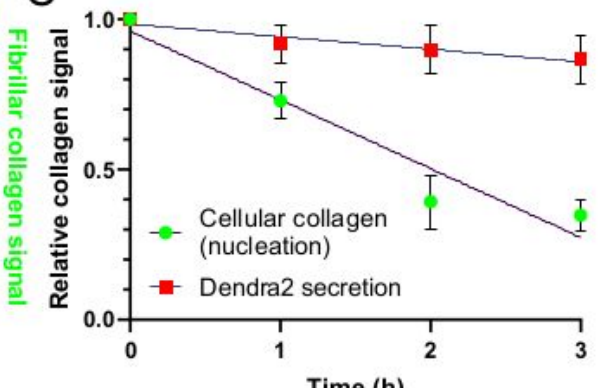

Time (h)
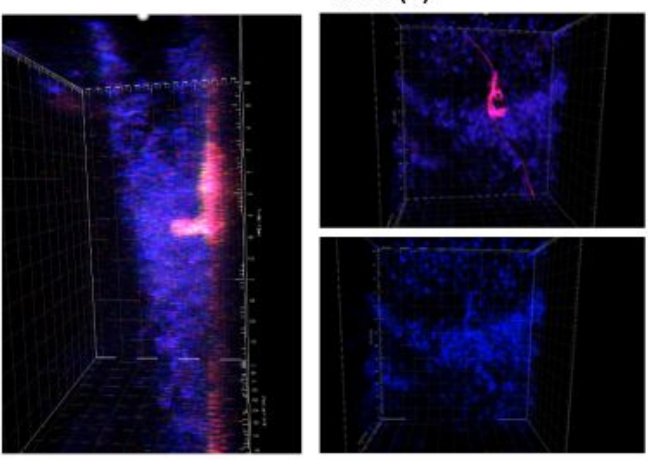

\section{Figure 2}

Cells control collagen fibrillogenesis. A) Airyscan microscopy of Dendra2::Col1a2 NIH3T3 cells demonstrating that Dendra2 positive fibrils are formed within the boundary of the cell body. Images were captured after $48 \mathrm{~h}$ in culture. The maximum intensity projection of 51 different $\mathrm{Z}$ positions are shown on the left, middle panel shows the localization of Dendra2::Col1a2 in a single Z plane within the cell body, right panel shows that Dendra2 fibrils are abundant on the cells basal surface, a single Z plane is shown. 
Arrows indicate Z position of each image. Video 2 shows all 51 positions. B) Quantification of cellular Dendra2::Col1a2 signal intensity in the cell body prior to the onset of fibril formation, termed nucleation, cellular Dendra2::Col1a2 signals drop dramatically during nucleation but then later recover as fibrils continue to grow. Nucleation was set at the frame prior to the first detection of Dendra2 positive fibrils, this was designated as $\mathrm{T}=0$, and the cellular Dendra2 signal was set as 1 , background pixel intensity was set as 0 . Data from five cells producing collagen fibrils are shown, data from individual cells are shown in Extended data figure 2. Average data points are shown, $n=5$ individual cells, error bars represent SD. C) The loss of cellular Dendra2 signal observed in B demonstrates rapid release of cellular collagen at the onset of fibril formation, the rate of loss is compared to the rate of loss of photoswitched Dendra2 signal in the absence of fibril formation (Figure 1D). Average data points are shown, $n=5$ individual cells, error bars represent SD. D) Expression of BFP-KDEL in Dendra2::Col1a2 NIH3T3 cells demonstrates that fibrils are formed on the basal surface of cells. A maximum intensity projection of $42 \mathrm{z}$ positions is shown, left. A 3D reconstruction of these cells show collagen fibrils are distinct from the cell body (Video 3), however loops in the collagen fibrils were observed extending into the cell body, a 3D reconstruction is shown in Video 3. The middle panel shows that photoswitched Dendra2 positive fibrils are beneath the cell body, importantly at positions of where loops are engulfed by the cell the endoplasmic reticulum (BFP-KDEL) align with the fibrils. 


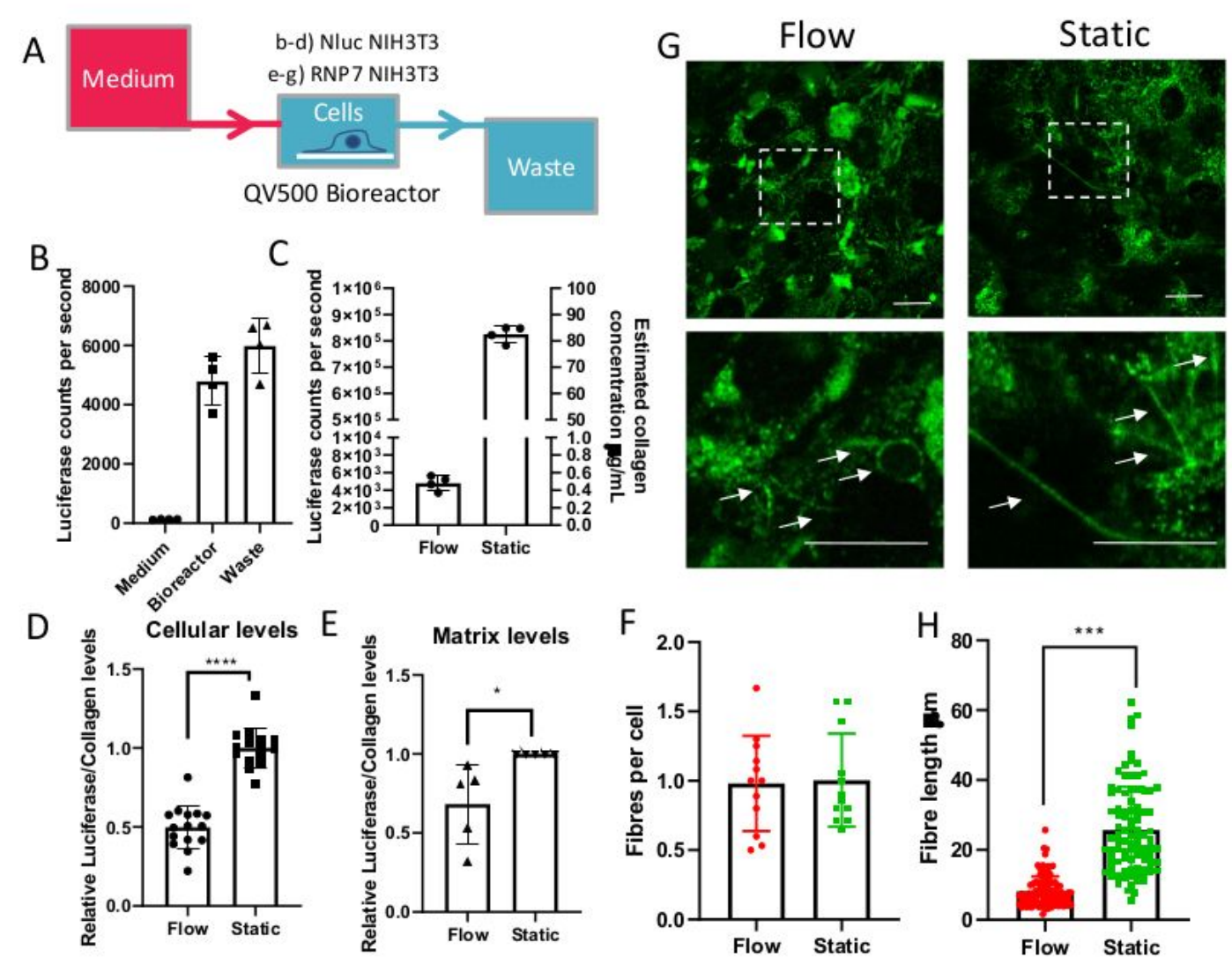

\section{Figure 3}

Rapidly secreted collagen contributes to fibril growth but not fibril nucleation. A) Schematic of CRISPRCas9 knock-in cells grown under constant flow. B) To validate the flow system Nluc::Col1a2 NIH3T3 cells were grown under flow. Nluc activity was assessed in the medium within the Bioreactor housing cells seeded on glass coverslips. The activity was also measured in the medium within the waste outflow ( $\mathrm{n}=4$ independent experiments, error bars show SD). C) Comparison of Nluc activity within the medium of 
Nluc::Col1a2 NIH3T3 cells grown under flow conditions or under static 'normal' culture conditions for 72 h. Comparison of Nluc activity in the medium was compared to recombinant Nluc protein to estimate the concentration of collagen within the medium as described previously (Calverley et al., 2020). D) After $72 \mathrm{~h}$ culture cells were removed from the bioreactor and subjected to assessment of cell viability and then coverslips were decellularized, measurements of Nluc activity in decellularized buffer were measured in triplicate ( $\mathrm{n}=4$ independent experiments, error bars show SD). 12 E) After decellularization, Nluc activity associated with the deposited matrix by cell grown under flow measured relative to cells grown under static conditions. $\mathrm{N}=5$ independent experiments, error bars show SD. F) The number of Dendra2 collagen fibrils per cell were scored, $n=3$ fields per view from 4 independent samples. G) Dendra2::Col1a2 NIH3T3 cells were grown under flow or static conditions for $72 \mathrm{~h}$. Airyscan microscopy of live cells identified Dendra2 positive fibrils. Scale bar represents $20 \mu \mathrm{m}$ ) H) The length of individual Dendra2 collagen fibrils were assessed using Image $\mathrm{J}$ image analysis software across all samples identified in $\mathrm{G}$. 


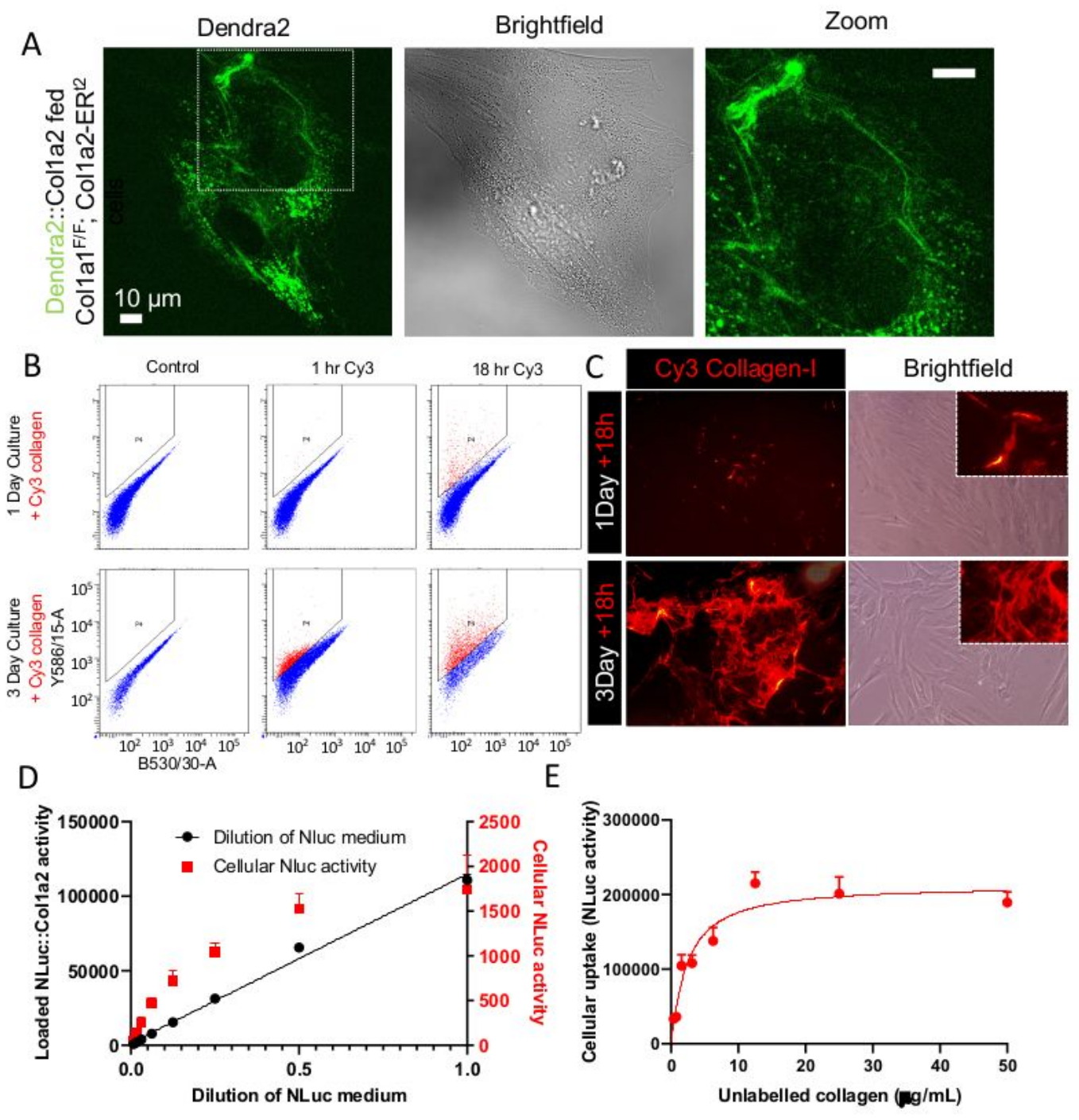

Figure 4

Endocytosed collagen contributes to fibril assembly. A) Fibroblasts isolated from tamoxifen treated Col1a1F/F; Col1a2-ER 82 t2 mice (Li et al., 2017; Yang et al., 2013) were fed with conditioned medium from Dendra2::Col1a2 NIH3T3 for $72 \mathrm{~h}$, medium was replaced each day. Dendra2 positive fibrils were also detected associated with cells. Scale bar represents $10 \mu \mathrm{m}$. B) $10 \mu \mathrm{g} / \mathrm{mL}$ Cy3 labeled rat tail collagen was added to cultures of human foreskin fibroblasts for 1 or $18 \mathrm{~h}$ demonstrating that fibroblasts were 
able to endocytose type-I collagen. Uptake was significantly enhanced in cells cultured for $72 \mathrm{~h}$ compared to those cultured for only $24 \mathrm{~h}$, see Extended data figure 3. C) Human foreskin fibroblasts fed with 0.5 $\mu \mathrm{g} / \mathrm{mL}$ Cy3 collagen for $18 \mathrm{~h}$ were effective at incorporating the labeled collagen into fibrillary structures whereas cells that were cultured for only $24 \mathrm{~h}$ were less effective in assembling fibrils. D) Tendon fibroblasts were fed with conditioned medium from Nluc::Col1a2 NIH3T3 cells. The amount of Nluc activity taken up by tendon fibroblasts after $18 \mathrm{~h}$ was measured, demonstrating that cells were able to endocytose Nluc::Col1a2, however there was a limit to the amount of Nluc activity that can be endocytosed. $\mathrm{N}=3$ independent experiments, error bars show SD. E) Tendon fibroblasts grown for $24 \mathrm{~h}$ were fed with Nluc::Col1a2 NIH3T3 conditioned medium along with increasing concentrations of rat tail collagen-I. After $18 \mathrm{~h}$ incubation, cellular Nluc activity was assessed. $\mathrm{N}=3$ independent experiments, error bars show SD. 


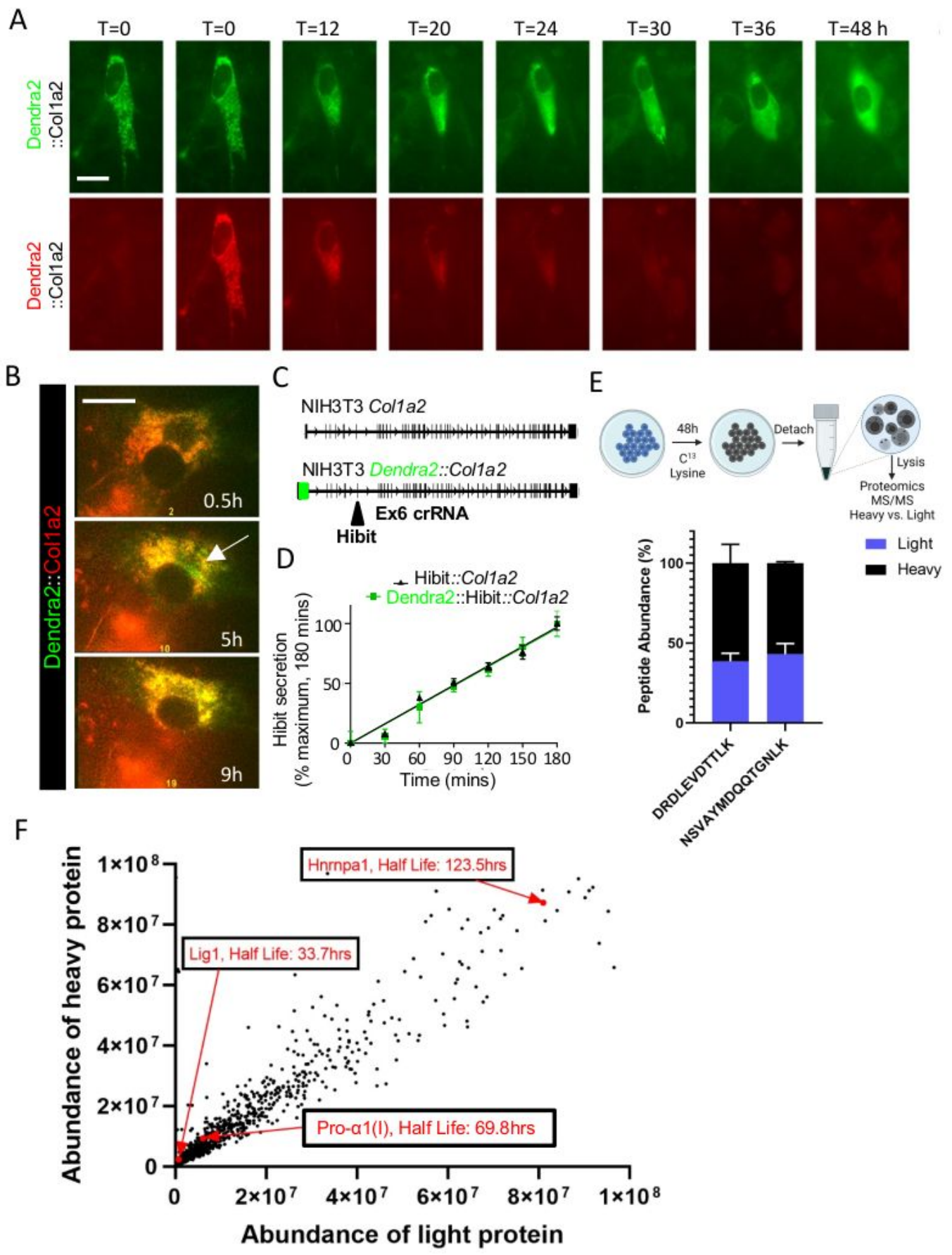

\section{Figure 5}

Secretion and storage of PCl. A. Selected frames from Video 1 showing that photoswitched Dendra2::Col1a2 (Red) has a longer life span within cells than predicted from Nluc::Col1a2 edited cells. Photoswitched collagen was detected intracellularly after $30 \mathrm{~h}$ post-switch. B. Selected frames from Extended data figure 4 showing transition of newly synthesized Dendra2::Col1a2 (displaying uniquely green fluorescence signal) through a Golgi-like structure. Concurrently pre-existing Dendra2::Col1a2 
remained detectable beyond $9 \mathrm{~h}$ post photoswitching. C. CRISPR-Cas 9 knock-in strategy to tag the amino telopeptide of proa2(I) with HiBit in unedited and Dendra2::Col1a2 cells within the region of Col1a2. HiBit is located $\mathrm{C}$-terminal of the $\mathrm{N}$ proteinase cleavage site and $\mathrm{N}$-terminal of the start of the major triple helical domain. This site is encoded by sequences within exon 6 of the Col1 a2 gene. D. Introduction of the split nanoluciferase sequence encoding HiBit was confirmed by detection of HiBit in the medium of edited cells. The rate of secretion for HiBit::Col1a2 and 13 Dendra2::Hibit::Col1a2 was measured as in Figure $1 A, n=4$ replicate measurements, error bars shown SD. E. To assess PCI lifetime in unedited $\mathrm{NIH} 3 \mathrm{~T} 3$ cells, cells were fed with $\mathrm{C} 13$ Lysine for $48 \mathrm{~h}$ before detachment, lysis and proteomic analysis. Peptide sequences shown are from the NC1 domain of proa1(I) mouse. Quantification of both heavy and light labeled Col1a1-derived peptides demonstrate that not all PCl is secreted within $48 \mathrm{~h}$. $\mathrm{N}=3$ independent experiments. F. Global protein abundance in SILAC labeled NIH3T3 cells, the indicated protein half-lives were estimated by measuring the abundance of both heavy and light peptides for each protein as described in Methods. 
A
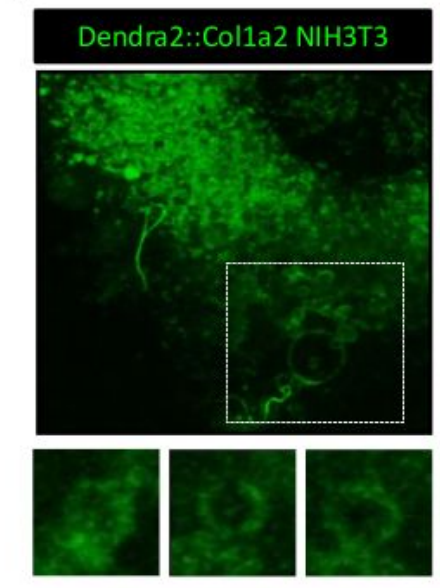

C

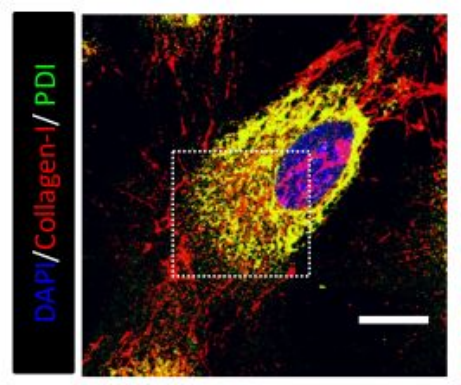

$E$
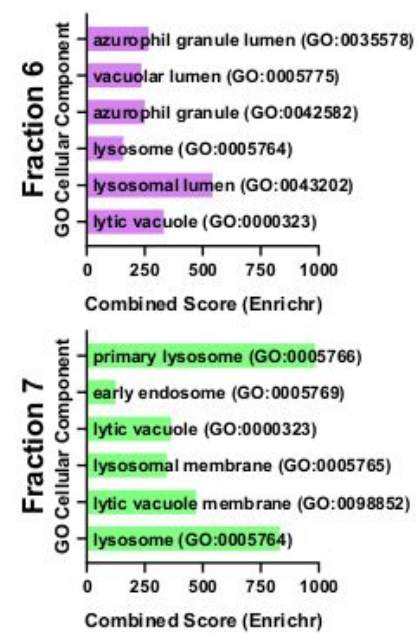

B
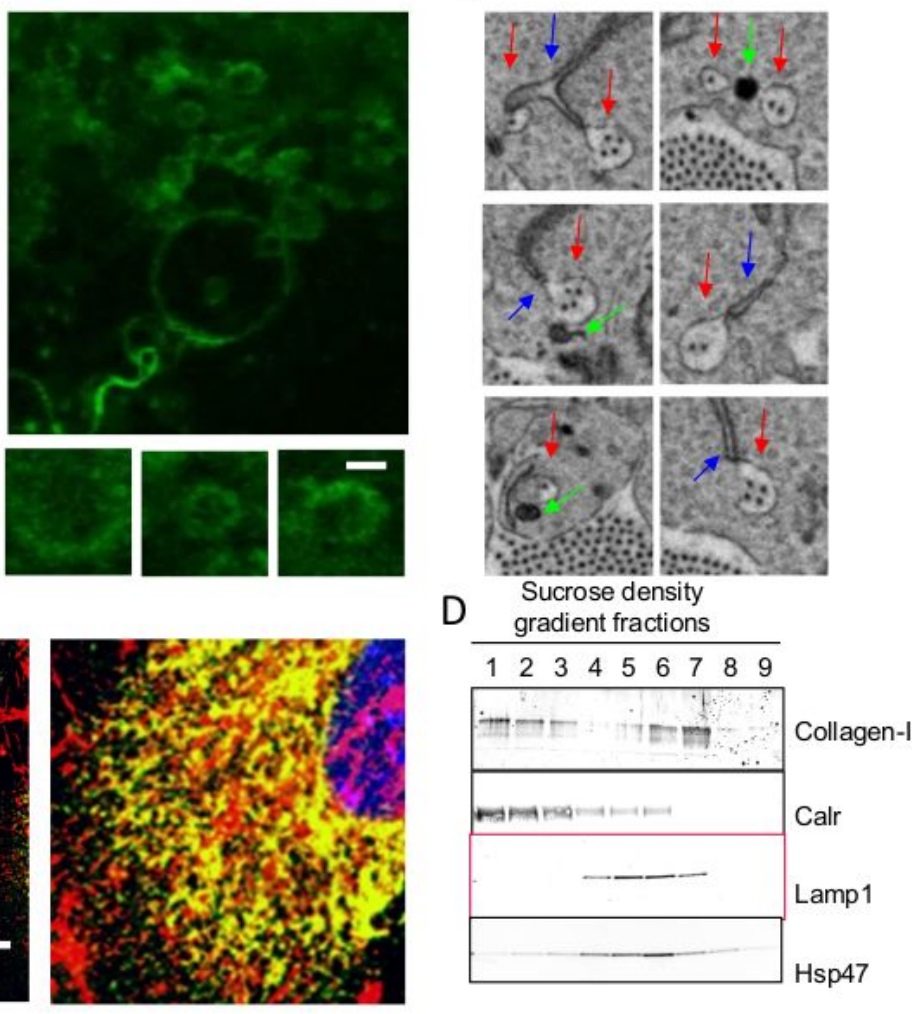

F
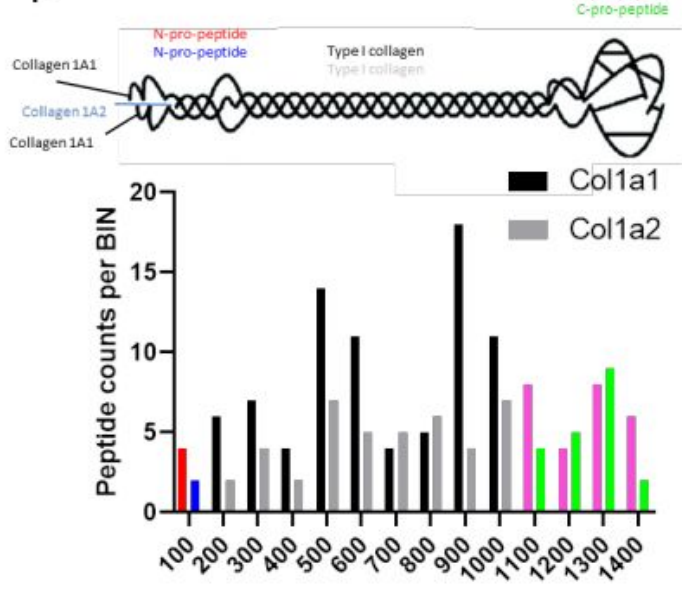

Peptide position aa (100aa BIN)

\section{Figure 6}

$\mathrm{PCl}$ abundant at sites of fibril formation. A. Dendra2::Col1a2 NIH3T3 imaged using Airyscan microscopy after $18 \mathrm{~h}$ after culture, $1000 \mathrm{x}$ magnification, and $2.5 \mathrm{x}$ digital zoom, a maximum intensity projection is shown. Vesicles containing Dendra2 signal are observed at sites of fibril assembly. Dendra2 signal was observed arranged at the periphery of vesicles, scale bar represents $1 \mu \mathrm{m}$. B. Electron micrograph of embryonic mouse tail tendon cells containing fibripositors (red), individual frames taken from 3view 
reconstructions shown in Video 6, larger images are shown in Extended data figure 5. Fibripositors are regularly contacted by endoplasmic reticulum (blue) and electron dense vesicles (green). Video 7 show that the Golgi apparatus does not contact fibripositor. C. Immunofluorescence imaging of type I collagen in mouse embryonic fibroblasts with the ER marker PDI. Scale bar represents $20 \mu \mathrm{m}$. D. Fractionation of NIH3T3 using a sucrose density gradient, type I collagen is co-resident with the ER protein Calreticulin (Calr) but also with the lysosomal protein Lamp1 and the collagen chaperone Hp47. E. Proteomic analysis of lysosomal fractions 6 and 7 identified significant enrichment of proteins identified by proteomic analysis of fractions 6 and 7 based on GO Cellular component terms. F. Col1a1 (Red and Magenta) and Col1a2 (Blue and Green) derived procollagen peptides were present within both fractions 6 and 7 suggesting that newly synthesized collagen transitions these compartments. 

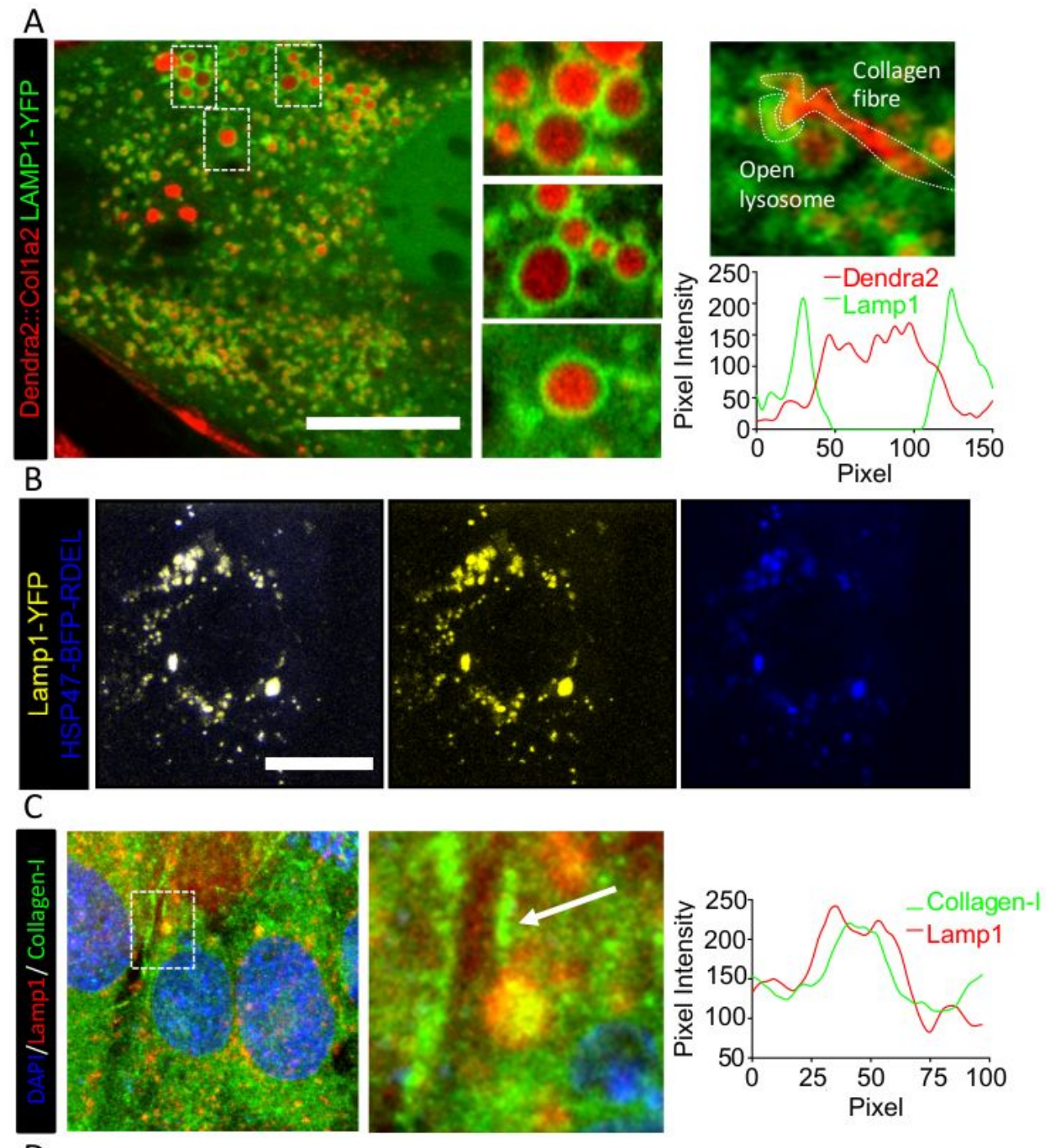

D
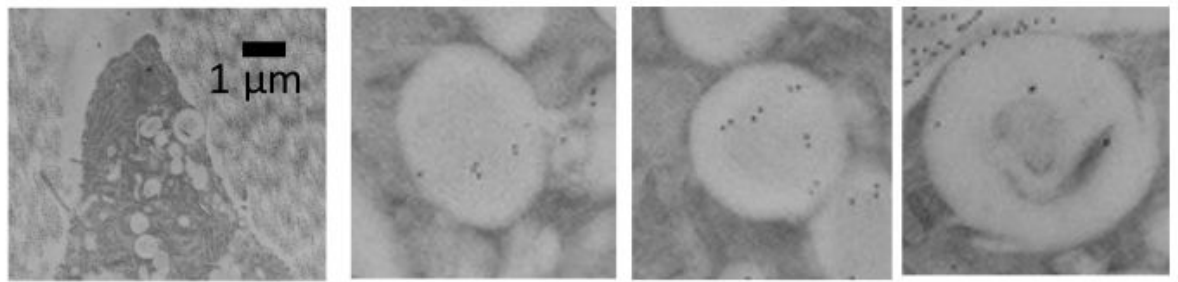

Figure 7

PCl localizes to LAMP1-positive compartments. A. Dendra2::Col1a2 NIH3T3 imaged using Airyscan microscopy $48 \mathrm{~h}$ after transfection with LAMP1-YFP. 1000x magnification, and 2.5x digital zoom, a maximum intensity projection of 31 images is shown. LAMP1-YFP vesicles containing photoswitched Dendra2 signals are observed. Three LAMP1-positive areas are enlarged. Using a single Z plane the pixel intensity of Dendra2 (Red) and LAMP1-YFP was measured in FIJI. At sites of fibril assembly LAMP1-YFP 
was observed juxtaposed to red Dendra2 fibrils. Scale bar represents $20 \mu \mathrm{m}$. B. Live cell super resolution microscopy of NIH3T3 stably transduced with Hp47-BFP-RDEL lentivirus and transfected with LAMP1YFP. Scale bar represents $20 \mu \mathrm{m}$. C. Immortalized tail tendon fibroblasts were grown in ascorbic acid containing medium for 3 days, cells were fixed and permeabilized and stained for collagen I, LAMP1 and counter stained with DAPI. A single optical section showing collagen I localization to LAMP1-positive lysosomes, a type I collagen fibril is indicated by the white arrow. Pixel intensity across the LAMP1positive vesicle is shown. $14 \mathrm{D}$. Immunogold electron microscopy labelling of collagen-1 of mouse tendon identifying extracellular collagen fibrils and intracellular collagen within hollow lumen vesicles, left, individual vesicles are enlarged. Scale bar represents $1 \mu \mathrm{m}$. 
A

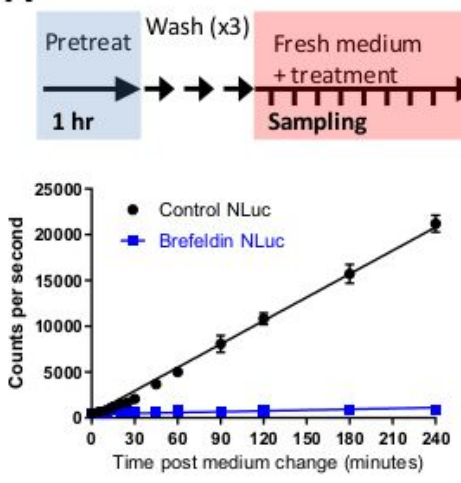

B

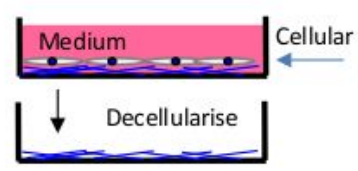

C

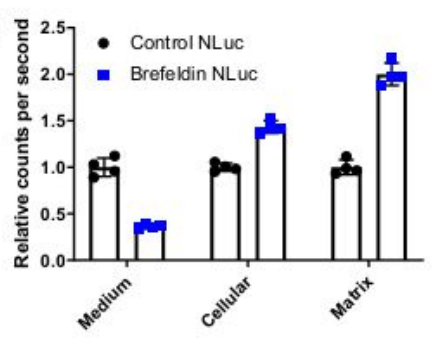

$\mathbf{G}_{\text {总悹 }}$

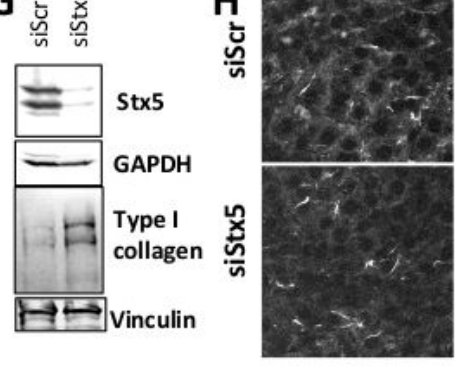

D
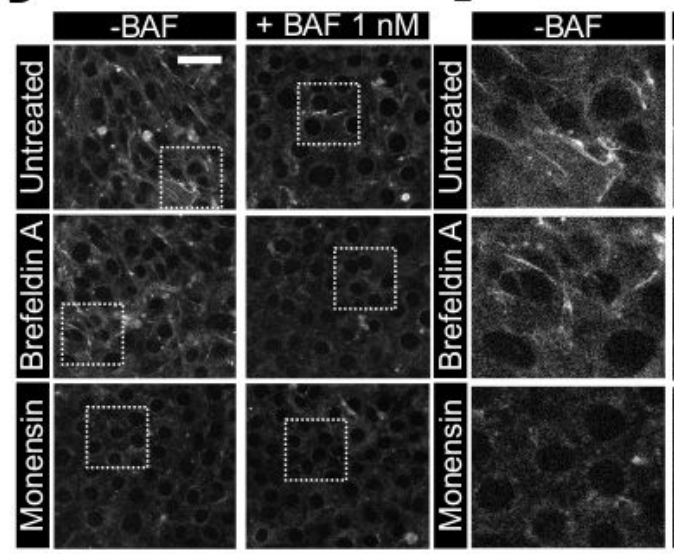

$+\mathrm{BAF} 1 \mathrm{nM}$
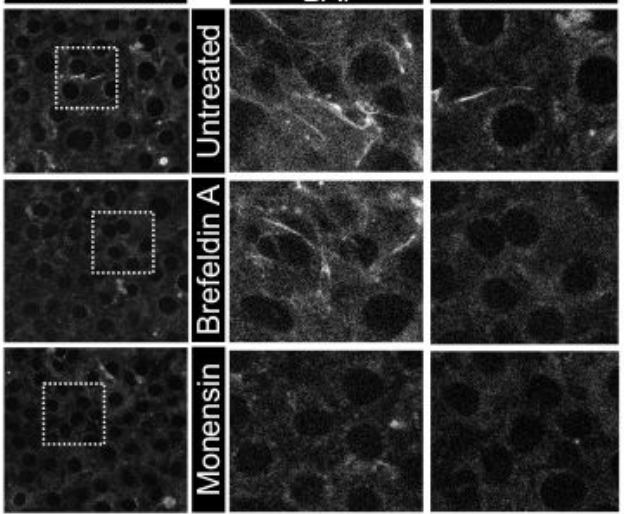

$\mathbf{F}$
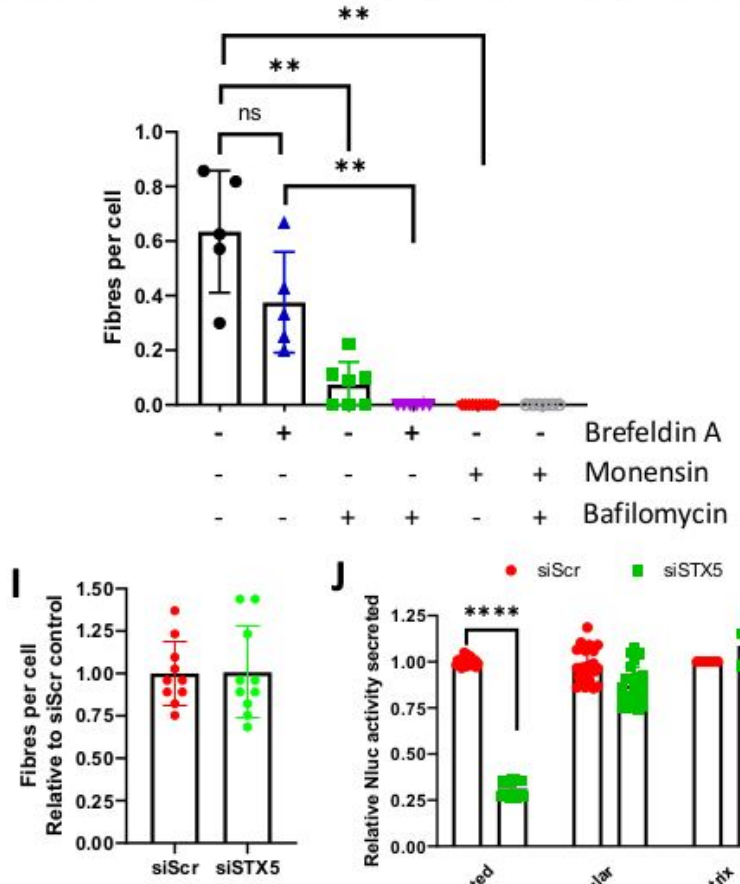

J.

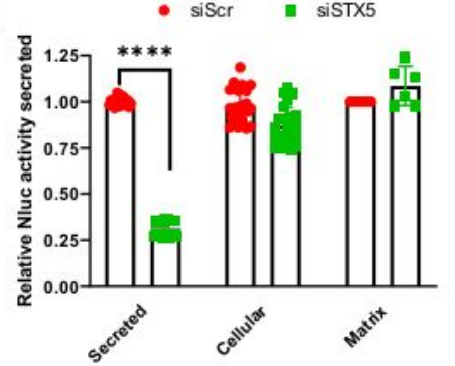

\section{Figure 8}

Non-classical PCl secretion feeds fibril assembly. A. Schematic for monitoring Nluc activity secretion from Nluc::Col1a2 NIH3T3 cells. Comparison of Nluc secretion rates in control cultures and cells treated with $100 \mathrm{nM}$ brefeldin A identified robust reduction of Nluc activity secretion. B. Schematic to quantify the deposition of Nluc::Col1a2 into the extracellular matrix. C. Nluc::Col1a2 NIH3T3 cells were cultured with $100 \mathrm{nM}$ brefeldin A for $72 \mathrm{~h}$, Nluc activity levels in the conditioned medium, cellular fraction and matrix 
were assessed. $\mathrm{N}=3$ independent experiments, $\mathrm{N}=4$ technical repeats from a representative experiment are shown. Error bars represent standard deviation. D. Representative Airyscan confocal microscopy images of Dendra2::Col1a2 NIH3T3 cells cultured for $72 \mathrm{~h}$ in the presence of either $100 \mathrm{nM}$ brefeldin $\mathrm{A}$, or $1 \mu \mathrm{M}$ monensin with or without addition of the lysosome proton pump inhibitor $1 \mathrm{nM}$ bafilomycin. Dendra2 signals are shown to highlight deposited fibrils. Maximum intensity projections of $5 \mathrm{z}$ planes are shown. Scale bar represents $50 \mu \mathrm{m}$. Boxes are enlarged in E. E. Enlargements of images within the regions highlighted in D. F. Quantification of Dendra2 fibril numbers per cell, $n=5$ independent experiments for brefeldin and monensin treatment, with an additional $n=2$ independent experiments for treatments including bafilomycin treatment. A minimum of 200 cells per condition, per experiment, were score. Error bars represent SEM. ** represents $p<0.01$, Student's T-test, unpaired. G. Western blot of Syntaxin 5 (Stx5) knockdown with 100 pmol siRNA in NIH3T3 cells. N=3 independent experiments. H. Syntaxin 5 (Stx5) knockdown with 100 pmol siRNA in Dendra2::Col1a2 NIH3T3 cells were cultured for $72 \mathrm{~h}$ before live imaging of Dendra2 fibrils. Maximum intensity projections of $5 \mathrm{z}$ planes are shown. Scale bar represents $50 \mu \mathrm{m}$. I. Quantification of Dendra2 fibril numbers per cell in siRNA treated Dendra2::Col1a2 NIH3T3. $n=2$ independent. Error bars represent SD. J. Syntaxin 5 (Stx5) knockdown with 100 pmol siRNA in Nluc::Col1a2 NIH3T3 cells were cultured for $72 \mathrm{~h}$, Nluc activity levels in the conditioned medium, cellular fraction and matrix were assessed. $\mathrm{N}=3$ independent experiments, each with $\mathrm{N}=4$ technical repeats are shown for conditioned medium and cellular fractions, or $n=2$ technical repeats for matrix measurements. Error bars represent standard deviation. 

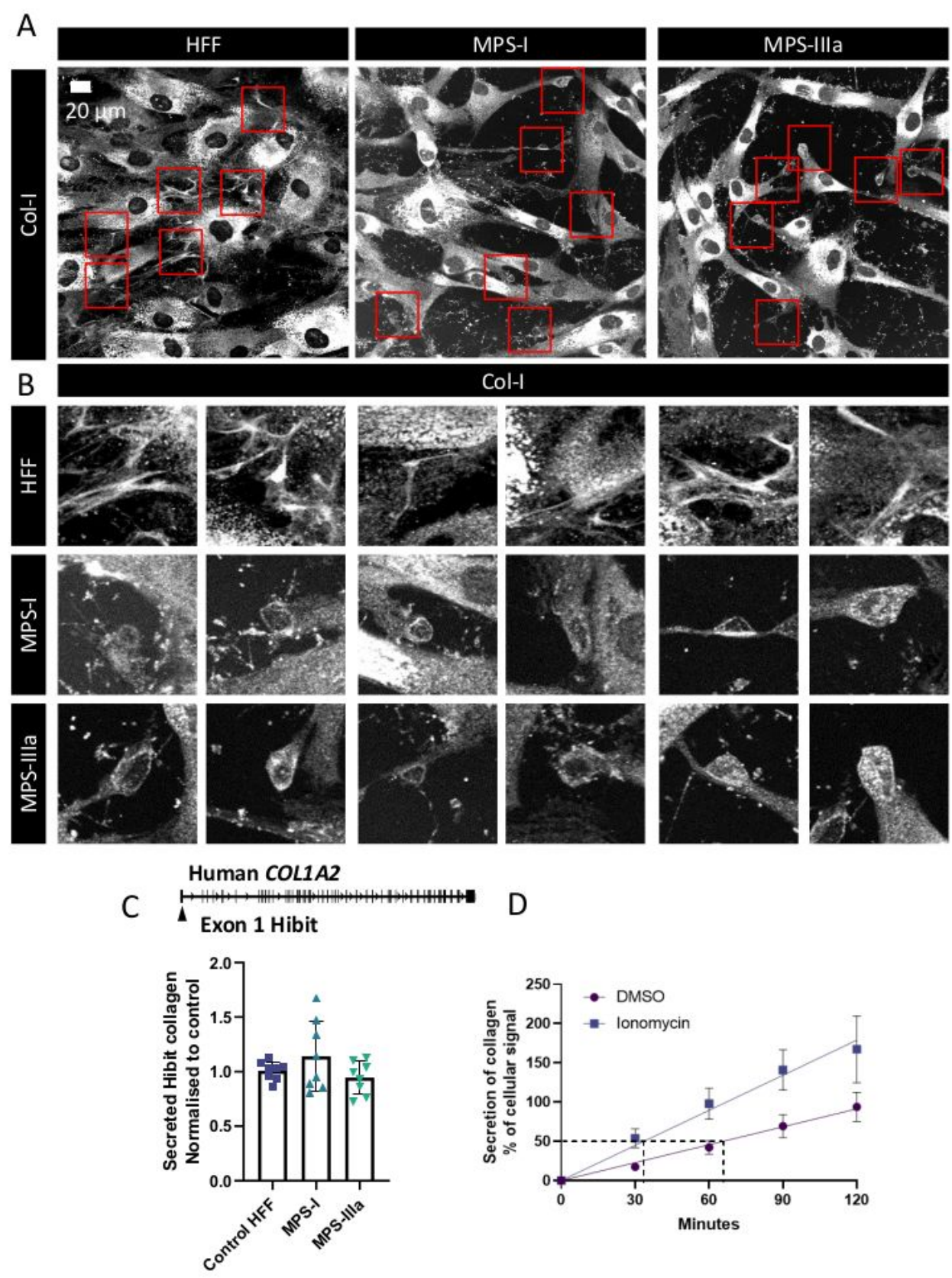

\section{Figure 9}

Disruption of type-I collagen fibril assembly in fibroblasts from patients with lysosome storage disorders. A) Immunofluorescence detection of type-I collagen in human skin fibroblasts taken from patients with lysosomal storage disorders, mucopolysaccharidosis type 1 and tpe $3 a$ (MPSI, and MPSIlla). Scale bars represent $20 \mu \mathrm{m}$. B) Regions highlighted in A show failed collagen assembly sites in MPS patient derived fibroblasts. C) CRISPR-Cas9 mediated knock-in of the split nanoluciferase tag, Hibit, into the Exon 1 of 
Col1a2 in control HFF and MPS patient derived fibroblasts. Secretion rates of Hibit tagged collagen 15 were normalized to cellular Hibit levels and indicated that there was no difference in the ability to secrete type-I collagen. D) Ionomycin triggered release of Nluc::Col1a2 from NIH3T3 cells. $\mathrm{N}=9$ independent experiments, error bars represent SEM.

\section{Supplementary Files}

This is a list of supplementary files associated with this preprint. Click to download.

- PickardVideo1.mp4

- PickardVideo3.avi

- PickardVideo6.mp4

- PickardExtendedDataFigure1.pdf

- PickardExtendedDataFigure2.pdf

- PickardExtendedDataFigure3.pdf

- PickardExtendedDataFigure4.pdf

- PickardExtendedDataFigure5.pdf

- PickardVideo2.mp4

- PickardExtendedDataFigure6.pdf

- PickardExtendedDataFigure7.pdf

- PickardVideo4.avi

- PickardSupplementaryTable1.xlsx

- PickardExtendedDataFigure8.pdf

- PickardSupplementaryTable2.xIsx

- PickardSupplementaryTable3.xlsx

- PickardSupplementaryTable4.xlsx

- PickardVideo7.avi

- PickardVideo5.avi 